San Jose State University

SJSU ScholarWorks

Master's Theses

Master's Theses and Graduate Research

2005

\title{
The effect of kinesthetic and spatial information in manual control performance
}

Arik-Quang V. Dao

San Jose State University

Follow this and additional works at: https://scholarworks.sjsu.edu/etd_theses

\section{Recommended Citation}

Dao, Arik-Quang V., "The effect of kinesthetic and spatial information in manual control performance" (2005). Master's Theses. 2754.

DOI: https://doi.org/10.31979/etd.348a-9sgu

https://scholarworks.sjsu.edu/etd_theses/2754

This Thesis is brought to you for free and open access by the Master's Theses and Graduate Research at SJSU ScholarWorks. It has been accepted for inclusion in Master's Theses by an authorized administrator of SJSU ScholarWorks. For more information, please contact scholarworks@sjsu.edu. 


\title{
THE EFFECT OF KINESTHETIC AND SPATIAL INFORMATION IN MANUAL CONTROL PERFORMANCE
}

\author{
A Thesis \\ Presented to \\ San Jose State University \\ In Partial Fulfillment \\ Of the Requirements for the Degree \\ Master of Science
}

The Faculty of the Graduate Program in Human Factors and Ergonomics

By

Arik-Quang V. Dao

August 2005 
UMI Number: 1429417

Copyright 2005 by

Dao, Arik-Quang V.

All rights reserved.

\section{INFORMATION TO USERS}

The quality of this reproduction is dependent upon the quality of the copy submitted. Broken or indistinct print, colored or poor quality illustrations and photographs, print bleed-through, substandard margins, and improper alignment can adversely affect reproduction.

In the unlikely event that the author did not send a complete manuscript and there are missing pages, these will be noted. Also, if unauthorized copyright material had to be removed, a note will indicate the deletion.

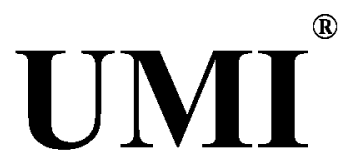

UMI Microform 1429417

Copyright 2006 by ProQuest Information and Learning Company. All rights reserved. This microform edition is protected against unauthorized copying under Title 17, United States Code.

ProQuest Information and Learning Company 300 North Zeeb Road

P.O. Box 1346

Ann Arbor, MI 48106-1346 
(C) 2005

Arik-Quang V. Dao

ALL RIGHTS RESERVED 
APPROVED FOR THE DEPARTMENT OF INDUSTRIAL

\section{SYSTEMS ENGINEERING}
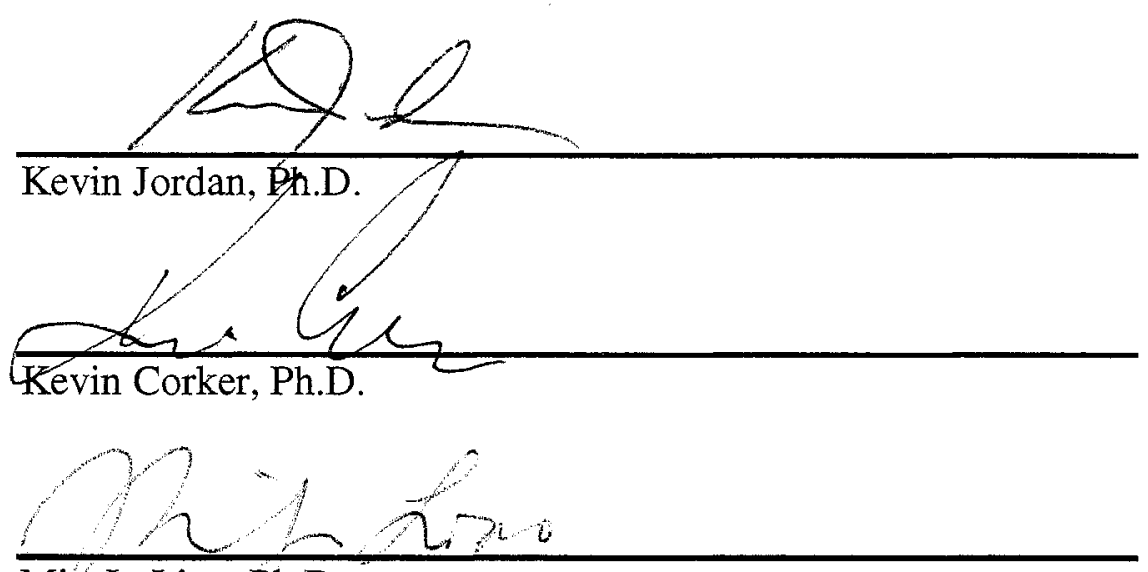

Min-Ju Liao, Ph.D.

APPROVED FOR THE UNIVERSITY

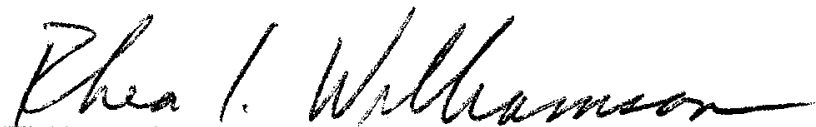




\title{
ABSTRACT \\ THE EFFECT OF KINESTHETIC AND SPATIAL INFORMATION IN MANUAL CONTROL PERFORMANCE
}

\author{
by Arik-Quang V. Dao
}

It has been shown that kinesthesia can be directly and indirectly manipulated to improve response times on various mental transformation and manual control tasks. In a target acquisition task, we varied symbol type (hand vs. arrow) and control type (kinesthetic, spatial, kinesthetic and spatial combined, and inconsistent) to investigate the effect of kinesthetic versus spatial information on manual control performance. A total of 32 participants were tested. Results of the study suggest that spatial information has greater influence on manual control performance than kinesthetic information. Implications of the findings are discussed in detail. 


\section{ACKNOWLEDGEMENTS}

I would like to extend my sincere gratitude to my respected teacher and mentor Dr. Min-Ju Liao, whose unyielding patience and resilience persisted at times when I just wasn't getting it; whose lessons extend beyond this endeavor and into life itself. Thank you to Dr. Kevin Jordan who, despite his intensively busy schedule, took on just one more responsibility, and whose contributions helped progress by leaps and bounds.

I am also indebted to Mr. Anthony Tang whose gifted programming skills made this study a reality. Thank you also to Professor Doreen Comerford-Roman who gave me the initial creative energy to drive my efforts over the steep intellectual threshold. Thank you to Mr. Vern Battiste and Dr. Walt Johnson for their contributions to this study, taking me in, and affording me opportunities when I was fresh out of undergrad. You have been generous to me. I would like to express my appreciation to Ms. Stacie "Witchcraft" Granada-Vigil whose dark magic brought supernatural improvement to my drafts during the final stretch. Your good humor reminded me that it is "all good" and that it gets better. Thanks also to the rest of the NASA Ames Flight Deck Display Research Laboratory team members, Mr. Riva Canton, Dominic Wong, and Tom Quinonez for enduring ramblings about my project during the group meetings. To Dr. Paul Lee who provided precious and spontaneous advice during visits to the break room making the cooler truly a fountain of knowledge. Thank you to Dr. Kevin Corker for a great program, whose inspiring lectures helped me to conceive my ideas.

Of course, I owe more than my life to my parents for providing me with a home environment of nourishment and source of replenishment when life's challenges have 
taken away the very last drop, and who has brought me up to have a taste and appreciation for the luxury of education. Your sacrifices and self-determination brought hope when luck had abandoned us. To my brother Minh Dao for "keeping things together" at home, while I've been out "joy reading." Lastly, thank you to Ms. Jan Yeung for listening. 


\section{TABLE OF CONTENTS}

SECTION

PAGE

INTRODUCTION

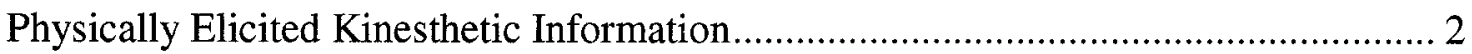

Motor Imagery: "Imagined” Kinesthesia .................................................................. 4

Influence of Visual Spatial Information ............................................................... 10

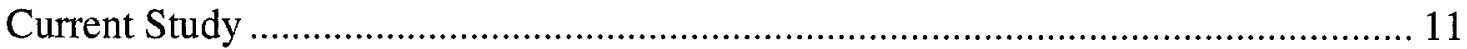

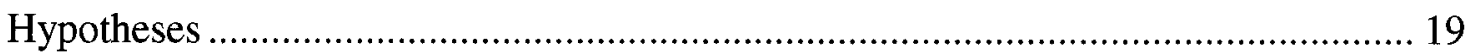

METHOD

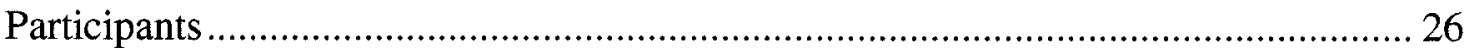

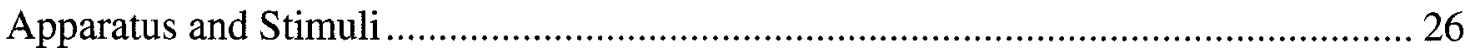

Control Mapping Conditions................................................................................. 29

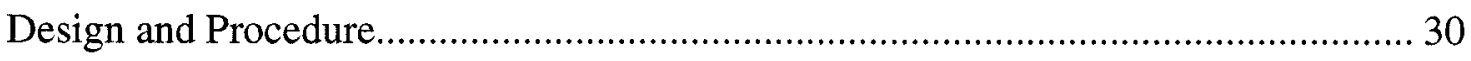

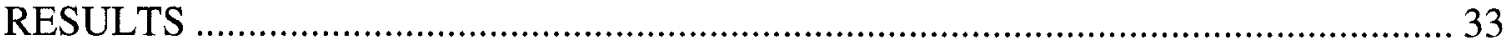

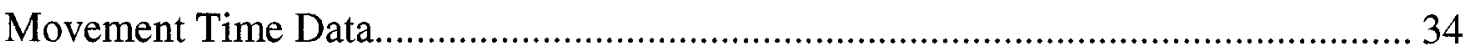

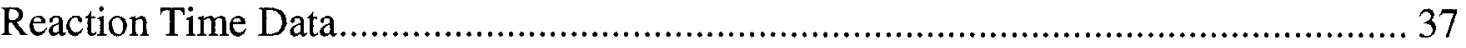

Self-report Data

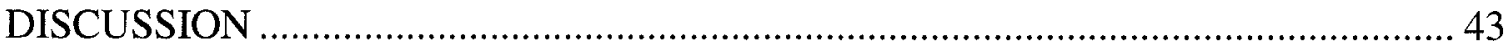

REFERENCES

APPENDIXES … -

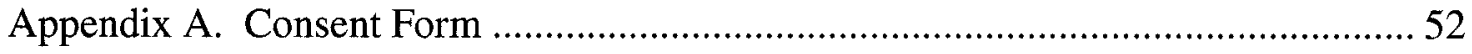

Appendix B. Human Subjects Approval Form.......................................................... 54 


\section{LIST OF TABLES}

TABLE

PAGE

1. Summary of responses collected from participant questionnaire. 


\section{LIST OF FIGURES}

FIGURE

PAGE

1. The use of left hand as kinesthetic cue for guiding right hand input control ................ 3

2. Five hand gesture variations used in Sekiyama's study ….......................................... 5

3. Mental rotation curves specific to particular hand gestures ........................................ 6

4. Mental rotation curve mirroring across left versus right handed gestures..................... 7

5. The effect of awareness of biomechanical constraints on mental rotation curve .......... 8

6. Orientation 1 and 2 for hand and arrow symbols .............................................. 12

7. In the kinesthetic mapping $(\mathrm{K})$ condition manipulating the control in the heave

direction moves the cursor to the right ............................................................ 14

8. In the spatial mapping (S) condition, heaving the control creates a direct spatial relationship by moving the cursor up along the $\mathrm{Y}$-axis ..................................... 15

9. In the kinesthetic and spatial combined (KS) mapping condition there is both spatial consistency in the control mapping relationship between the input device and the cursor, providing spatial information ....................................................... 16

10. In the inconsistent mapping (I) condition the spatially inconsistent control mapping

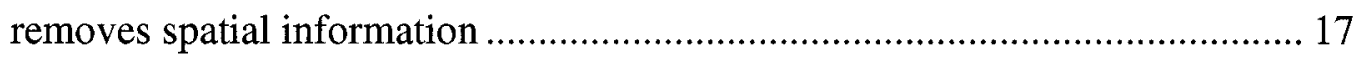

11. Predicted movement and reaction time patterns for each symbol type across four control mapping conditions, assuming that kinesthetic information will have an

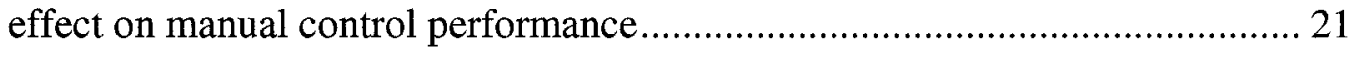

12. Predicted performance pattern across control mapping type collapsed over symbol type assuming an influence of kinesthetic information .................................... 22 
13. Predicted movement and reaction time patterns for each symbol type across four control mapping conditions, assuming that spatial information will have a greater influence on manual control performance

14. Predicted performance pattern across control mapping type collapsed over symbol type assuming that spatial information has a greater influence.

15. 3D Space Mouse

16. Task Frames. Participants were asked to view the cross hair in Frame 1 for 1 second, after which either the hand of arrow symbol appeared for an additional duration of 1 second 31

17. Mean movement times in milliseconds across four mapping conditions for each symbol type

18. Mean reaction time. Findings for reaction time were similar to those for movement time, again, demonstrating a greater influence of spatial information in manual control performance 39

19. Average age across control mapping conditions 40 


\section{INTRODUCTION}

Kinesthesia is the awareness or sense of our body position and movements (Bastian, 1888; McCloskey, 1978). Britton, Lipscomb, and Pique (1978) reported in a case study that providing kinesthetic information through kinesthetic correspondent mapping improved performance. Kinesthetic correspondent mapping was defined to have existed if a controlled image moves in the same direction of the hand manipulating the control device. In the aforementioned case study, 27 chemists used a kinesthetically mapped device called the GRIP-75 to determine the shapes of large molecules. The GRIP-75 was used in combination with 3D graphical molecular visualization software to match molecular stick figures to images of respective electron density shapes. From 1700 hours of interaction with the molecular visualization tools over a period of 2.5 years the chemists expressed that the kinesthetically correspondent mapping eased their work. It was from this subjective reporting that Britton et al. suggested that the kinesthetic information provided through the correspondent mapping improved productivity. However, Britton et al.'s case study had yet to provide objective data demonstrating that kinesthetic information improved manual control performance. Therefore, the purpose of this study was to examine, under controlled conditions, the role of kinesthetic and spatial information in manual control performance. In the following discussion, evidence will be presented that illustrates how physically eliciting kinesthetic information can improve manual control performance. In addition, the following sections will describe how 
studies using the mental rotation paradigm have shown that kinesthetic information can be elicited through visual imagery.

Physically Elicited Kinesthetic Information

In support of the idea that kinesthetic information augments manual control performance (Britton et al., 1978), Adelstein, Ellis, Smith, and Welch (2004) generated objective data demonstrating that such performance improvements can be achieved in the presence of a kinesthetic cue. Participants in Adelstein et al.'s study were composed of strongly right-handed male and female subjects. These participants were asked to acquire a target using their right hand to control a cursor using a trackpad or a trackball, and their left hand as a kinesthetic cue. When the input-to-display rotation was off-set, the left hand index finger (oriented around a block) was used to indicate the straight-ahead input direction, and the thumb would be used to indicate the horizontal right on the display (Figure 1). In an analysis of path motion, Adelstein et al. found that participants were more efficient when they were provided a kinesthetic cue when compared to conditions without a kinesthetic cue. Thus, it appears that manual control performance can be improved by directly providing the operator with kinesthetic information. However, Adelstein et al. focused mainly on the control domain and no attention was given to the possible interacting effects of display and control. That is, it is possible that depending upon the image type, visual stimuli can also be a source of kinesthetic information. The following section discusses this idea in detail. 


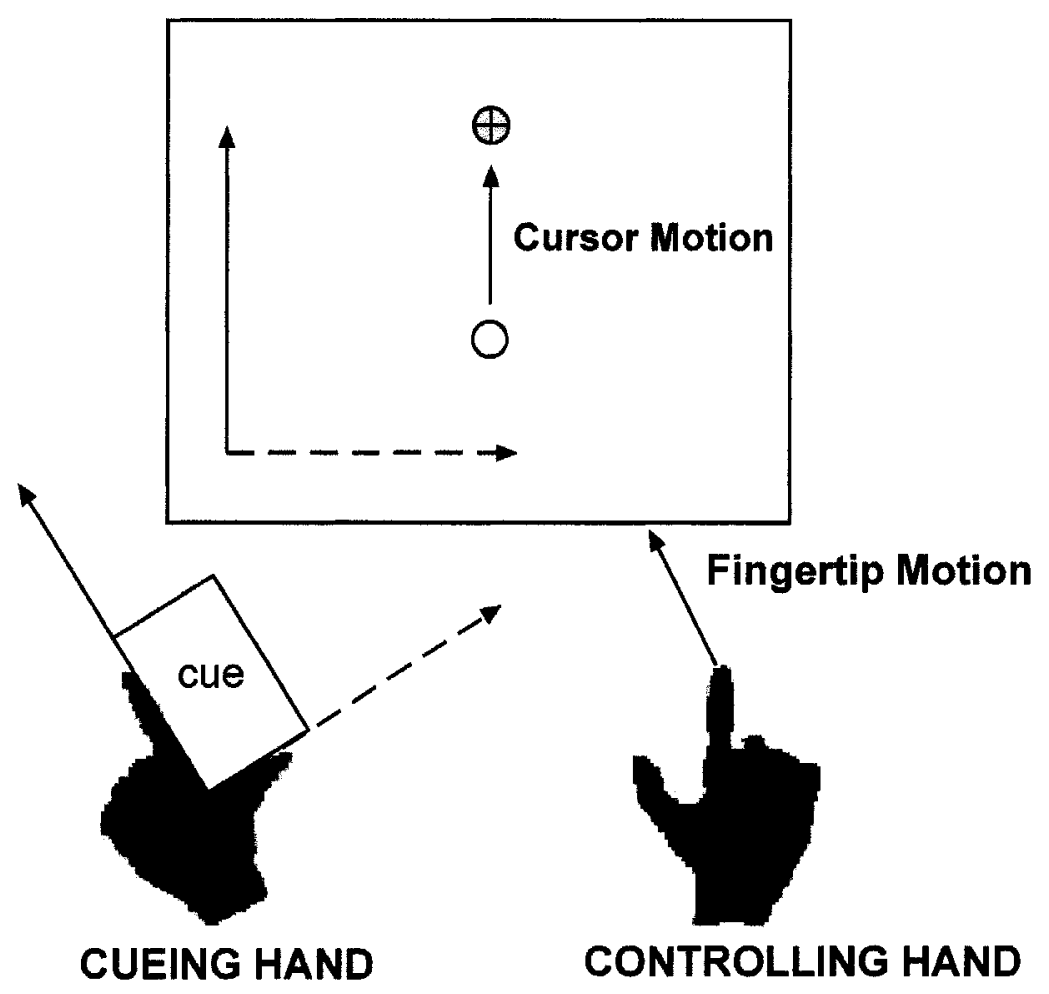

Figure 1. The use of left hand as kinesthetic cue for guiding right hand input control.

The index finger of the left hand revealed the change in the corresponding movements between the controlling hand and the cursor motion. 


\section{Motor Imagery: "Imagined" Kinesthesia}

Sekiyama (1982) examined the role of kinesthesia in mental rotation performance, participants were asked to determine if a figure of a particular hand gesture was a left or right hand by pressing a left or right handed button. A figure of a right hand was drawn in five different gestures (Figure 2). Another set of the same hand gestures were generated using a left handed configuration. The hand figures were then presented to participants at various degrees of departure $(45,90,135,180,270$, and 360 degrees). The notion that the hand gestures were visually eliciting kinesthesia was shown in three ways. One, each hand gesture elicited its own mental rotation data pattern (Figure 3). Second, there was a mirror reversed relationship between data patterns for left hand figures versus the right hand figures (Figure 4). Third, the typically symmetrical mental rotation curve which peaks at 180 degrees shifted based on the type of hand gesture. For example, in Figure 5, at 180 degrees it is difficult to tell which direction participants had mentally rotated to complete the task. However, because the data peaks at 135 degrees, it was reasoned that participants mentally rotated the image to the left. Although it would have been faster and more efficient for participants to rotate to the right, it appears that the kinesthetic effect of the visual stimuli gave participants the sense that rotating to the right would not be manageable due to physical constraints that would be imposed if the image was to be the participant's actual hand. The mentally imposed constraints that led participants to rotate toward a more biomechanically recognizable direction were what Sekiyama called a manageable direction of movement. Sekiyama's findings 


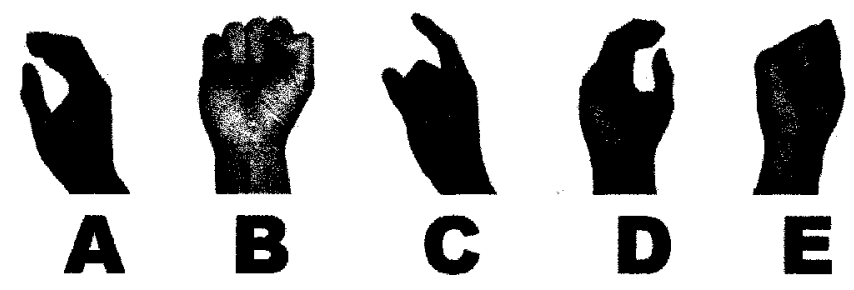

Figure 2. Five hand gesture variations used in Sekiyama's study. 


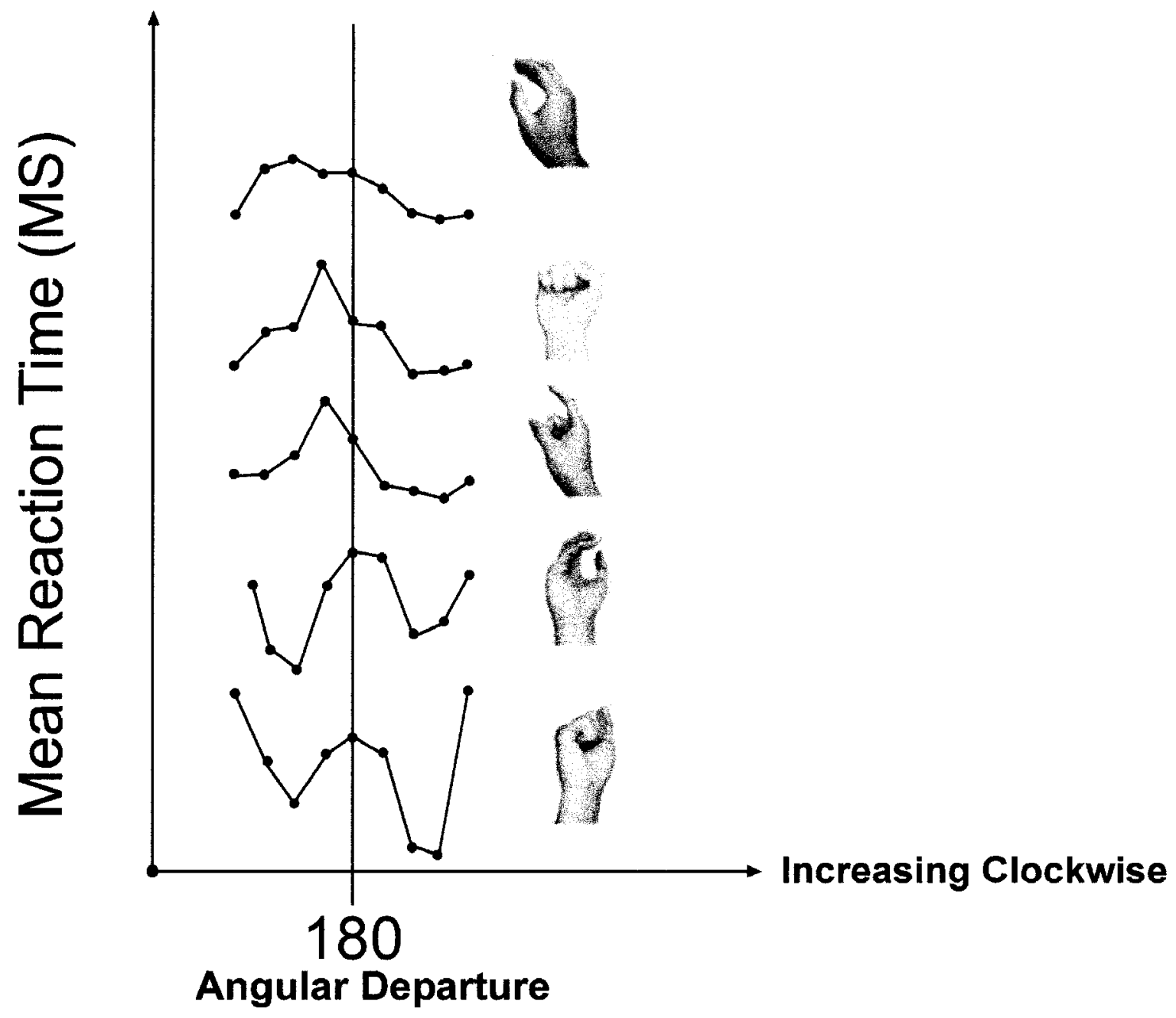

Figure 3. Mental rotation curves specific to particular hand gestures. The variation in mental rotation pattern across hand gesture type provides evidence of an "imagined" kinesthetic effect on mental transformation processes. 


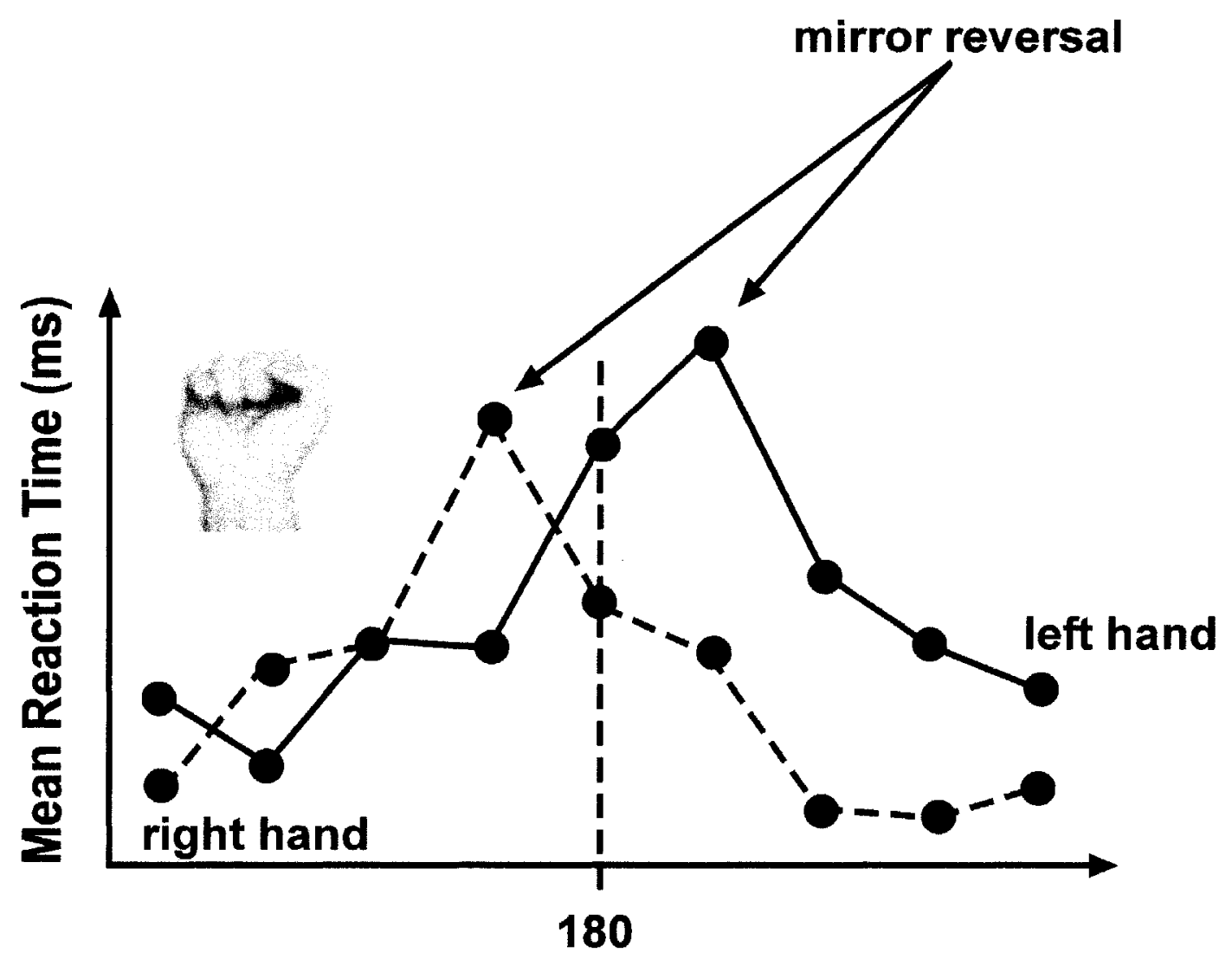

Figure 4. Mental rotation curve mirroring across left versus right handed gestures.

Observed mental rotation patterns were mirrored across left and right hand representations of each particular hand gesture, providing additional evidence of an effect of "imagined" kinesthesia. 


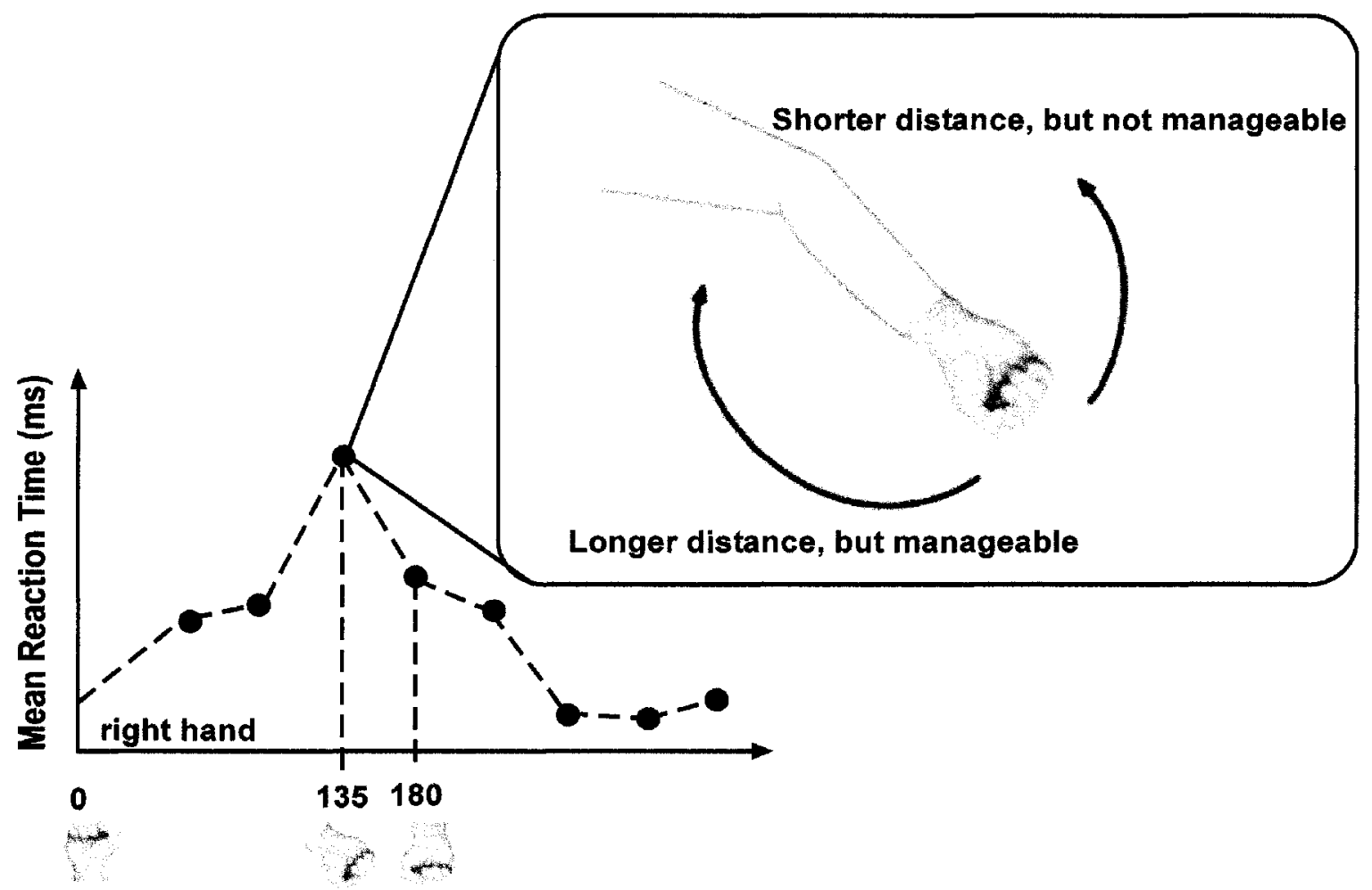

Figure 5. The effect of awareness of biomechanical constraints on mental rotation curve. Classic mental rotation curves peak at 180 degrees. In the figure above, the curve peaks at 135 degrees because participants imagined not being able to rotate the hand to the right (a relatively shorter distance) due to an awareness of the biomechanical constraints of the arm. Instead, participants preferred to rotate the arm to the left which was a longer distance, but a more manageable direction of movement. 
demonstrated that an awareness of the physical constraints of one's own hand can have an effect on the task of mentally rotating various hand gesture symbols.

This "imagined" kinesthetic awareness of our body, or motor imagery, has been demonstrated using the mental chronometric paradigm and has shown that the duration of “imagined" actions were proportional to actual actions (Crammond, 1997; Decety \& Jeannerod, 1995). For example, when participants were asked to determine if a picture of hand was left or right, when compared to conditions where no prior instruction was given, response times were faster when participants were asked to imagine their own hand superimposed on the displayed hand figure (Cooper \& Shepard, 1975). In another study, participants used their own body as a reference when asked to determine if a figure of a body rotated at varying degrees of departure was drawn with a left or right hand raised (Zacks, Mires, Tversky, \& Hazeltine, in press). Additional evidence of "imagined" kinesthesia comes from findings from a study conducted by Decety and Jeannerod (1995) which showed that the constraints on movement time described by Fitt's Law persisted when participants were asked to imagine and report the start and finish times of walking to increasingly narrow gates.

Consistent with chronometric studies, data collected from neurophysiological studies suggested that mental rotation involving body parts versus objects alone activate specific regions of the brain (Kosslyn, Thompson, Wraga, \& Alpert, 2001; Wolbers, Weiller, \& Buchel, 2003). Wolbers, Weiller, and Buchel (2003) demonstrated that when participants mentally rotated objects, visual spatial regions of the brain were activated. In contrast, when participants imagined rotating the same objects with their hands, 
somatosensory, as well as visual spatial regions of the brain showed pronounced activity. In light of the aforementioned studies, a kinesthetic influence on mental rotation would ultimately have implications on manual control performance due to the perceptual processing of visual stimuli being controlled. This importance of image type on eliciting such "imagined" kinesthesia and the possibility that it may interact with physical kinesthetic information provided in the control domain was explored in the current study. Influence of Visual Spatial Information

In order to understand the relationship between kinesthetic information and mapping as implied by Britton et al. (1978), it is necessary to consider mental transformation in the context of mental rotation tasks used in other studies. In the classic mental rotation paradigm, participants are asked to identify alphanumeric symbols or figures displayed at varying degrees departing from their normal upright presentation (Cooper and Shepard, 1973; 1975). Mental rotation is defined by an increase in reaction time as a function of an increasing degree of stimuli misorientation. During mental transformation, image rotation and the rotation of an internal frame of reference have been identified as mutually exclusive events (Zacks et al., in press). Image rotation is said to have occurred when participants identify symbols or words that have been misoriented by first visually rotating the stimuli to an upright position, or orientation that most closely matches the representation stored in memory. In the case of rotating an internal frame of reference, instead of rotating the image itself, perceivers mentally orient their own view into congruence with the misoriented stimuli. A study conducted by Jordan and Huntsman (1990) presented participants with words as well as non-words at 
varying degrees of misorientation and demonstrated that images are rotated rather than an internal frame of reference during mental transformation processes. In such a case, it would appear that mental transformation processes rely primarily on visual information, particularly if the image is a depiction of words or objects. The findings from Jordan and Huntsman (1990) reveal that spatial information may have an influence on mental transformation processes related to manual control performance. Therefore, in the current study, one goal is to examine manual control performance when kinesthetic information is present versus when kinesthetic information is absent, but spatial information is present.

\section{Current Study}

Recall that kinesthesia can be elicited by viewing images of body parts and that the same sense of kinesthesia may be absent when perceivers are presented with images of objects. To examine this image interdependency effect of kinesthesia we will compare performance data for figures of a hand versus an arrow at various orientations. It is important to note that our definition of a kinesthetic mapping differs from the kinesthetic correspondent mapping described by Britton et al. (1978) in that we will take into consideration the type and orientation of the image. Thus, for the purpose of this study, control mapping provided kinesthetic information when the direction of the movement of the hand or arrow symbol being controlled is consistent with the movement of the real hand. For example, provided that the hand symbol is in Orientation 1 (Figure 6) a heave (Y-axis) motion on a manual input device would correspond with movement of the cursor 


\section{Orientation 1}
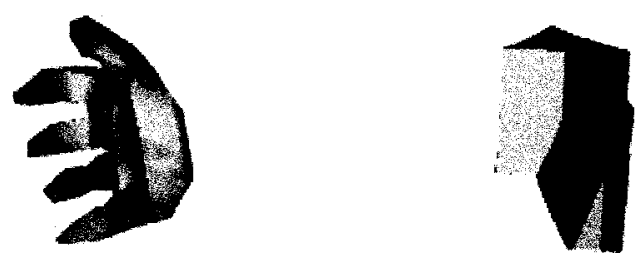

\section{Orientation 2}
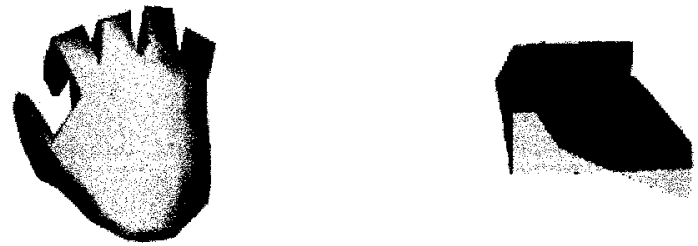

Figure 6. Orientation 1 and 2 for hand and arrow symbols. The independent variable symbol was consisted of two levels (hand vs. arrow). Each of these symbols were presented in one of two orientation. The orientation of the symbol determined the presence or absence of kinesthetic or spatial information. 
along the $\mathrm{X}$-axis. Thus, although the spatial relationship between the control and the hand cursor is inconsistent, the movement is consistent with the orientation of the hand cursor and the position of the hand controlling the mouse. We suggest that the consistency of the direction of movement for the hand operating the control and the cursor provides kinesthetic information (Figure 7).

Control mapping provides spatial information when the direction of the movement of the hand or arrow symbol being controlled is consistent with movement in space. When the input device was manipulated in the heave (Y-axis) direction, the cursor responds by moving up along the Y-axis. In the case illustrated in Figure 8, the movement of the hand cursor is consistent with the movement of the control in space, but inconsistent with the orientation of the cursor. In the kinesthetic/spatial mapping condition, where both spatial and kinesthetic information is present, both kinesthetic and spatial mapping correspond to symbol movement on both $\mathrm{X}$ and $\mathrm{Y}$-axis, and the cursor is in Orientation 2 (Figure 9). This way, the relationship of the movement between the input device and the hand cursor is spatially consistent, but also kinesthetically consistent because the hand is now oriented to match the controlling hand directly.

The baseline inconsistent mapping condition was generated when the movement of the cursor did not correspond kinesthetically or spatially. For example, when the Space Mouse was manipulated in the heave direction and the cursor was in Orientation 2, the cursor would respond by moving along the $\mathrm{X}$-axis (Figure 10). In the inconsistent mapping condition, spatial information is absent because of the inverse relationship between the movement of the input device and cursor, and kinesthetic information is 


\section{Cursor moves to the right or left along the $\mathrm{X}$-axis}
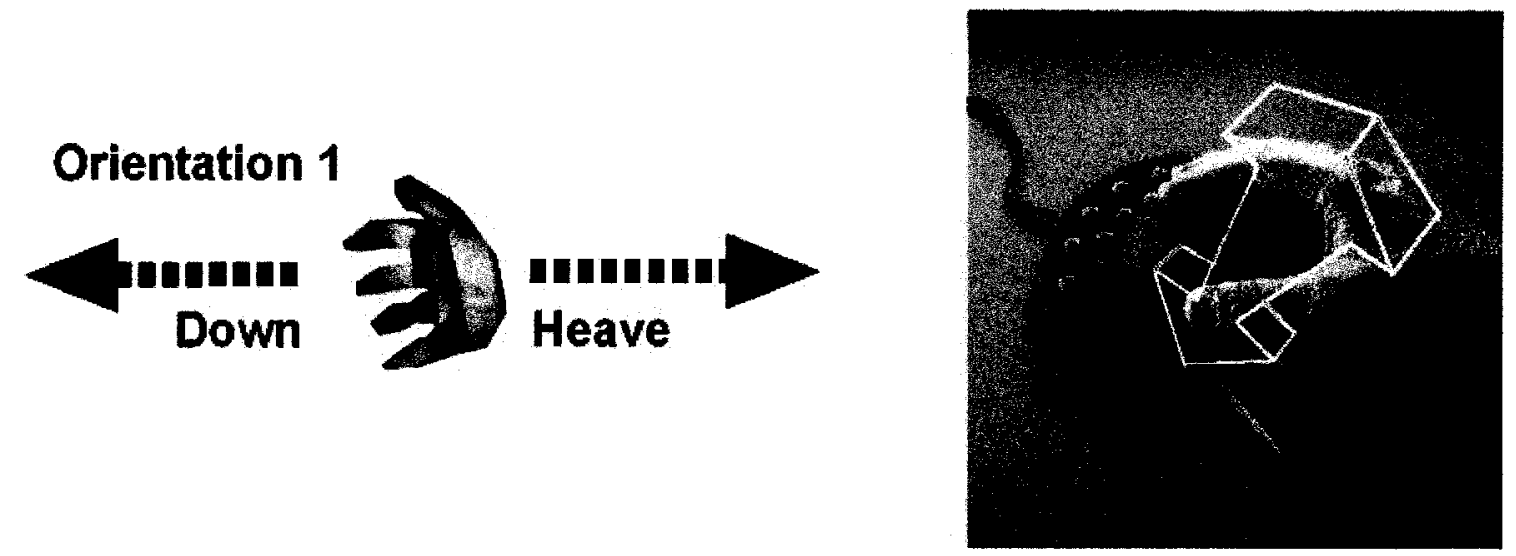

Figure 7. In the kinesthetic mapping $(\mathrm{K})$ condition manipulating the control in the heave (Y-axis) direction moves the cursor to the right. Pressing down on the control, moves the cursor to the left along the $\mathrm{X}$-axis. Here, the control mapping relationship between the cursor and the control is spatially inconsistent. However, the orientation of the cursor makes the control mapping kinesthetically consistent. Thus, this condition provides kinesthetic information when the controlling hand and cursor are in motion. 


\section{Cursor moves to the top or bottom of display along $Y$-axis}

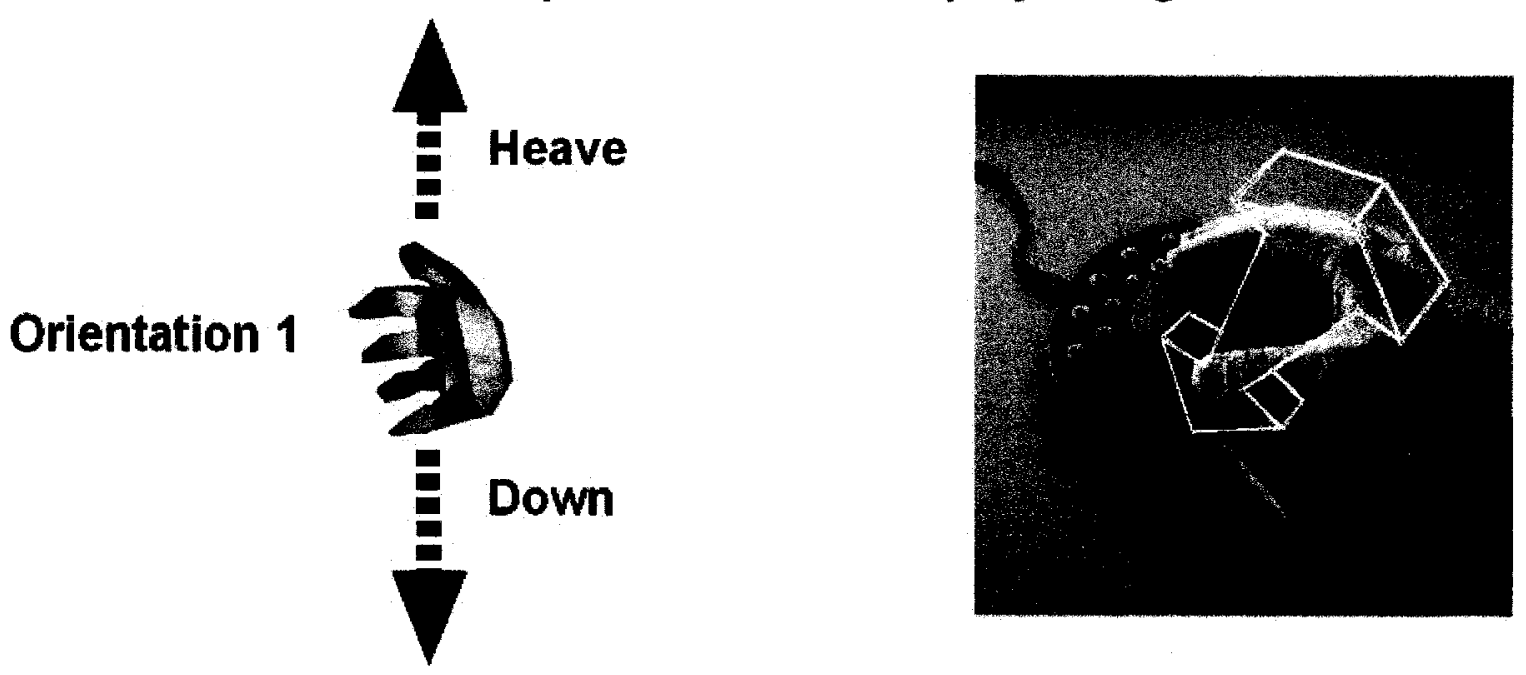

Figure 8. In the spatial mapping (S) condition, heaving the control creates a direct spatial relationship by moving the cursor up along the $\mathrm{Y}$-axis. Pushing down, moves the cursor down. Both the orientation of the cursor and the controlling hand are inconsistent, removing kinesthetic information. However, the control mapping is spatially consistent with the cursor direction of movement, providing only spatial information. 


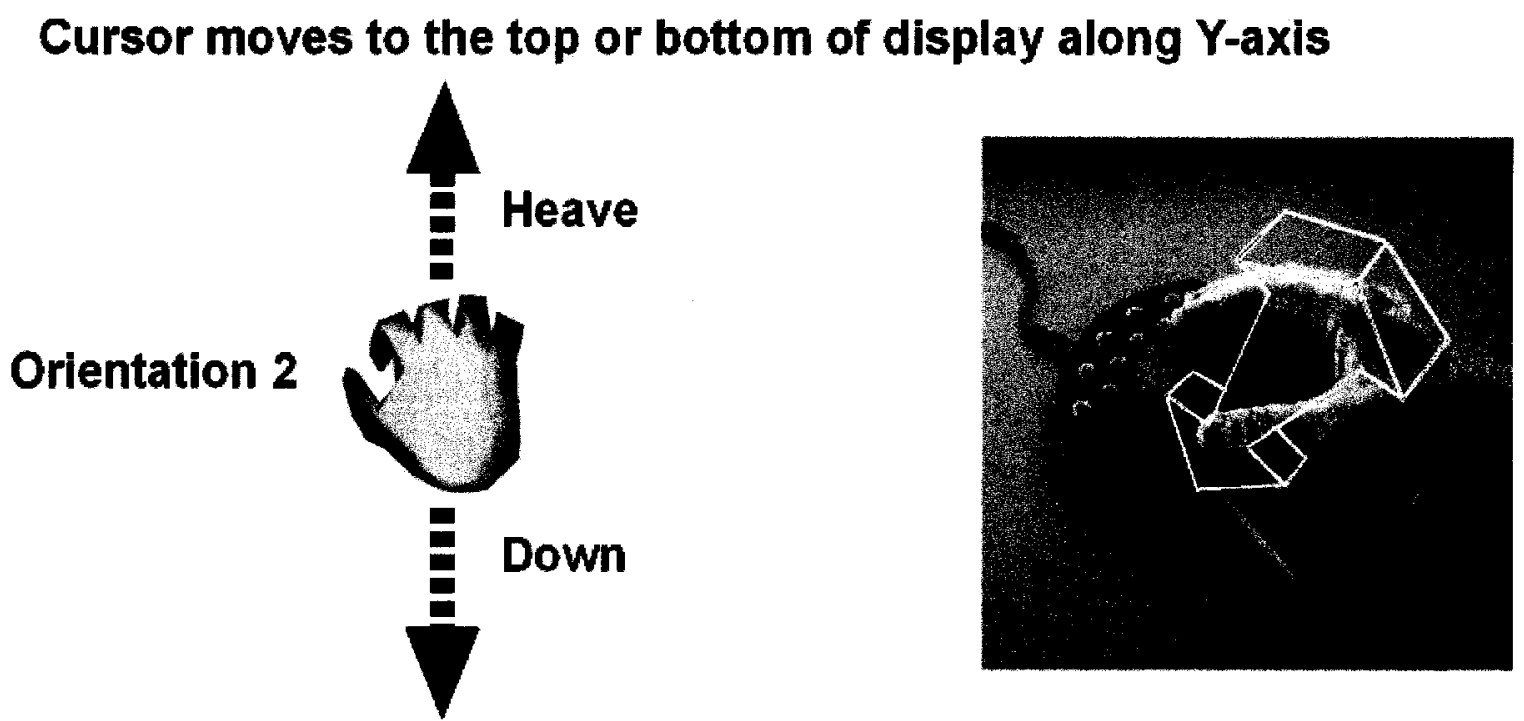

Figure 9. In the kinesthetic and spatial combined (KS) mapping condition there is both spatial consistency in the control mapping relationship between the input device and the cursor, providing spatial information. The KS condition also provides kinesthetic information because the spatial mapping and orientation of the cursor now makes all the cursor and hand movements kinesthetically consistent. 


\section{Cursor moves to the left or right of display along $\mathrm{X}$-axis}
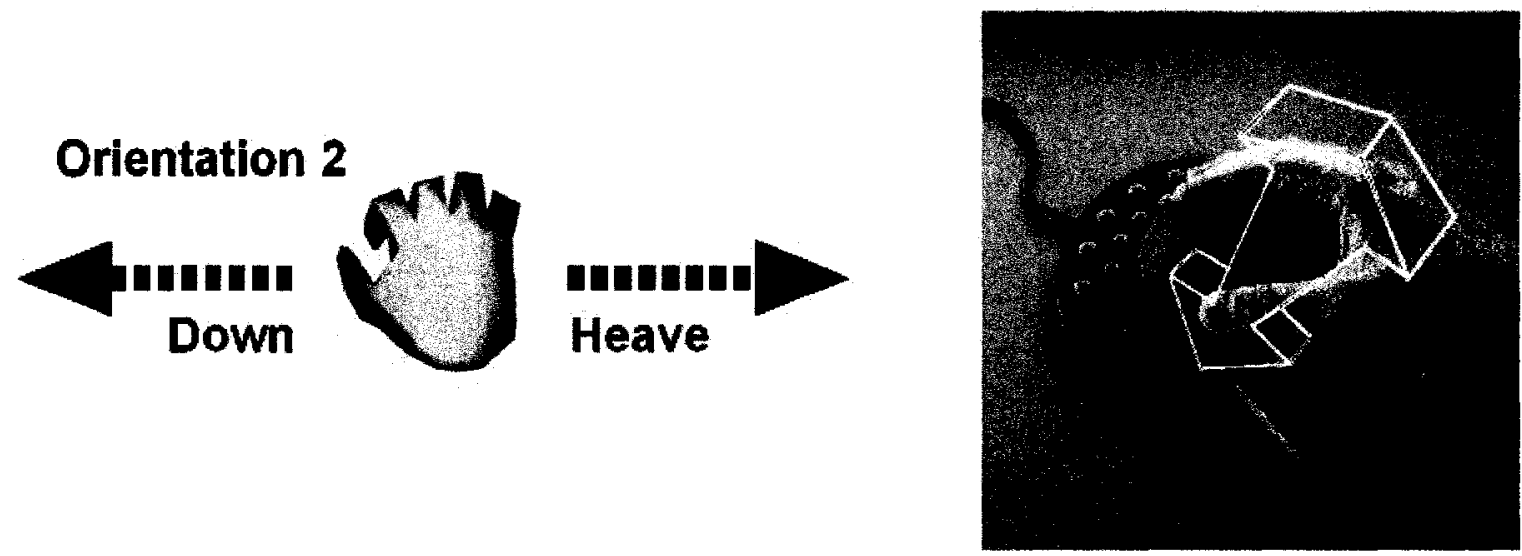

Figure 10. In the inconsistent mapping (I) condition the spatially inconsistent control mapping removes spatial information. In addition, the inconsistent control and the orientation of the cursor remove kinesthetic information. 
absent because the orientation of the hand cursor is not consistent with the direction of movement of the controlling hand.

Based on the previously mentioned mapping conditions one can see how defining kinesthetic correspondent mapping by configuring the cursor to move in the same direction of the hand (Britton et al., 1978) may lead to difficulty in differentiating kinesthetic from spatially mapped controls. Thus, it was necessary to modify the definition of kinesthetic correspondent mapping for the present study.

It is reasonable to suggest based on findings from Adelstein et al. (2004) and Sekiyama's (1982) studies that kinesthetic information may enhance manual control performance, favoring kinesthetic mapping. However, performance with the use of spatial information must also be considered because vision may dominate the processes underlying manual control performance, as shown in various mental rotation studies focused on visually related stimuli (Cooper \& Shepard, 1973; Jordan \& Huntsman, 1990). It is important to note that the aforementioned mental rotation studies did not include motor movement as a variable. The conditions used in those studies were purely visual (e.g., mentally rotated shapes, numbers, and words) generating data that would inherently give more weight to visual information in explaining mental rotation processes. In contrast, the current study will assess the contribution of motion as a contributor of spatial information. A final issue that was considered in the current study was that a combination of kinesthetic and spatial mapping may be preferred as it takes into consideration both spatial and kinesthetic information. The goal of the present study was to extend Sekiyama's (1982) study by examining the effect of object images versus 
the kinesthetic effect that may be specific to hand figures in four different control mapping conditions. In addition, we extended the study conducted by Adelstein et al. (2004) from the control domain by exploring the interacting effects of image type and control mapping.

\section{Hypotheses}

The following five hypotheses were generated provided that visual images can stimulate kinesthesia as shown in Sekiyama's study (1982). 1.) Although Sekiyama was able to demonstrate the presence of kinesthesia using still images, we did not predict a main effect for symbol type. We reasoned that unlike in previous studies which manipulated the orientation of the hand symbol, movements of the control are required in order for kinesthetic or spatial information to emerge in our examination of manual control performance. Thus, instead, we predicted an interaction effect between symbol type and control mapping type, and the performance patterns are described as follows. 2.) We expected that movement time would be faster for hand figures in the kinesthetic mapping condition, when compared to arrows, because the hand figures would have a greater kinesthetic effect. 3.) Although objects may elicit slower movement times in the kinesthetic mapping condition it is expected that they would elicit faster movement times in the spatially mapped manual control condition. 4.) We also hypothesize that both arrow and hand figure will elicit comparable movement times in the kinesthetic and spatially combined mapping condition because the mapping in this condition is expected to be optimal for all image types. 5.) The slowest performance was expected when participants were presented with an inconsistent mapping condition in which the cursor 
will provide absolutely no cueing with regards to cursor and control correspondence (Figure 11). We do expect a main effect across control mapping type in which the differences between symbol type will average out in the kinesthetic and spatial mapping conditions (Figure 12). However, relatively slower performance times would be observed in the inconsistent mapping conditions. In contrast, the fastest performance times would be observed when times are averaged across both image types in the kinesthetic and spatially combined mapping condition because, again, we expected this condition to be consistent kinesthetically and spatially.

Five hypotheses were generated assuming that there is no interaction effect of image type and control mapping type suggesting that hand figures would not be conducive to better performance on kinesthetically mapped controls. The following performance patterns were predicted (Figure 13). 1.) Both arrow and hand figure will exhibit comparably slower movement times in the kinesthetic mapping condition. 2.) Both the arrow and hand figure will exhibit comparably faster movement times in the spatially mapped control condition. 3.) Again, both arrow and hand figure will show comparably faster movement time in the kinesthetic and spatially combined mapping condition relative to kinesthetic and spatial mapping conditions alone. 4.) We expect that although both the hand and arrow cursors provide spatial information in this situation, the inconsistent control mapping situation would not be consistent with the spatially perceived cursors. Thus, performance was expected to be the most degraded in the inconsistent mapping condition. 5.) A main effect of control mapping would still be obtained when scores are averaged across symbol type. However, if spatial information 


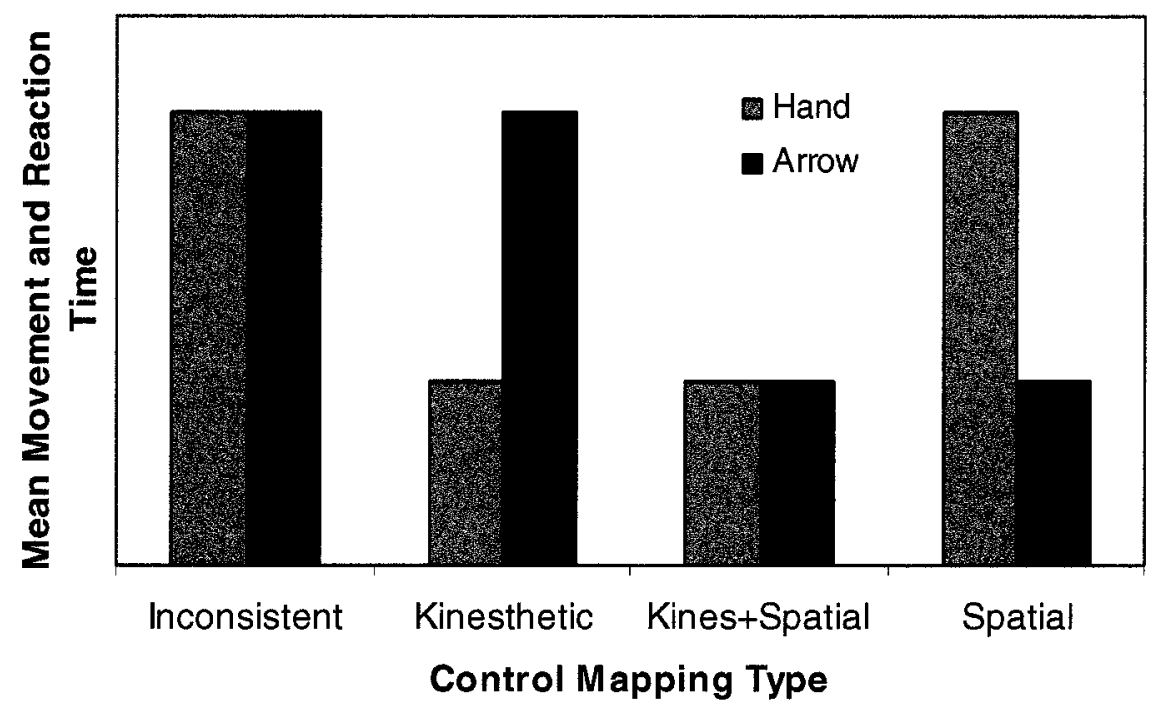

Figure 11. Predicted movement and reaction time patterns for each symbol type across four control mapping conditions, assuming that kinesthetic information will have an effect on manual control performance. 


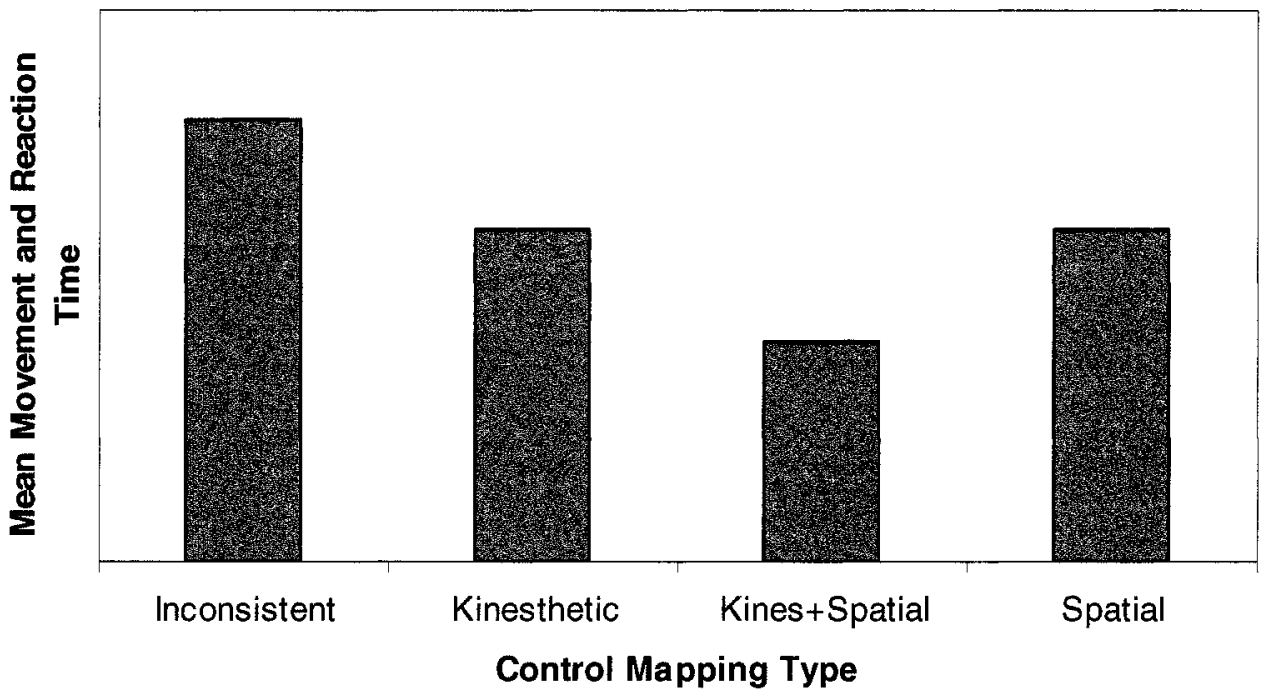

Figure 12. Predicted performance pattern across control mapping type collapsed over symbol type assuming an influence of kinesthetic information. 


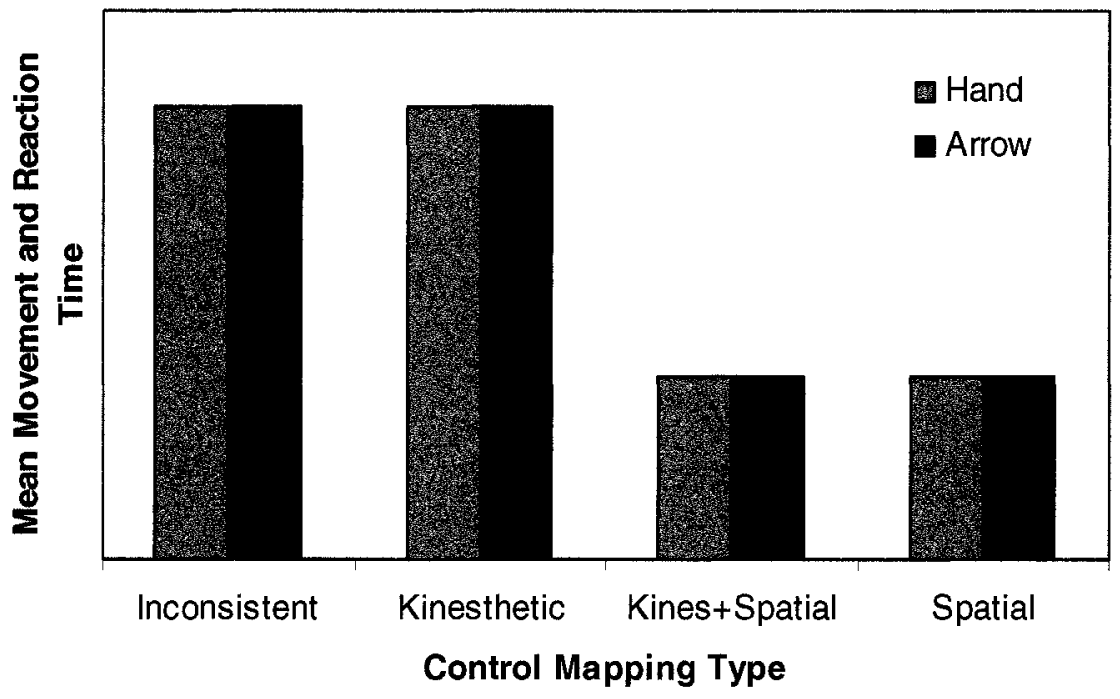

Figure 13. Predicted movement and reaction time patterns for each symbol type across four control mapping conditions, assuming that spatial information will have a greater influence on manual control performance. If spatial information plays a larger role than "imagined" kinesthetic information, it would be expected that significant differences would be observed mainly between control mapping types. A main effect of symbol type would not be present. 
has a greater influence on manual control performance, then we expect that the inconsistent and kinesthetic mapping conditions would elicit comparably slower performance times. In contrast, the spatial as well as kinesthetic and spatially combined mapping conditions would elicit comparably faster performance times because the control and cursor mapping relationship is spatially consistent (Figure 14). 


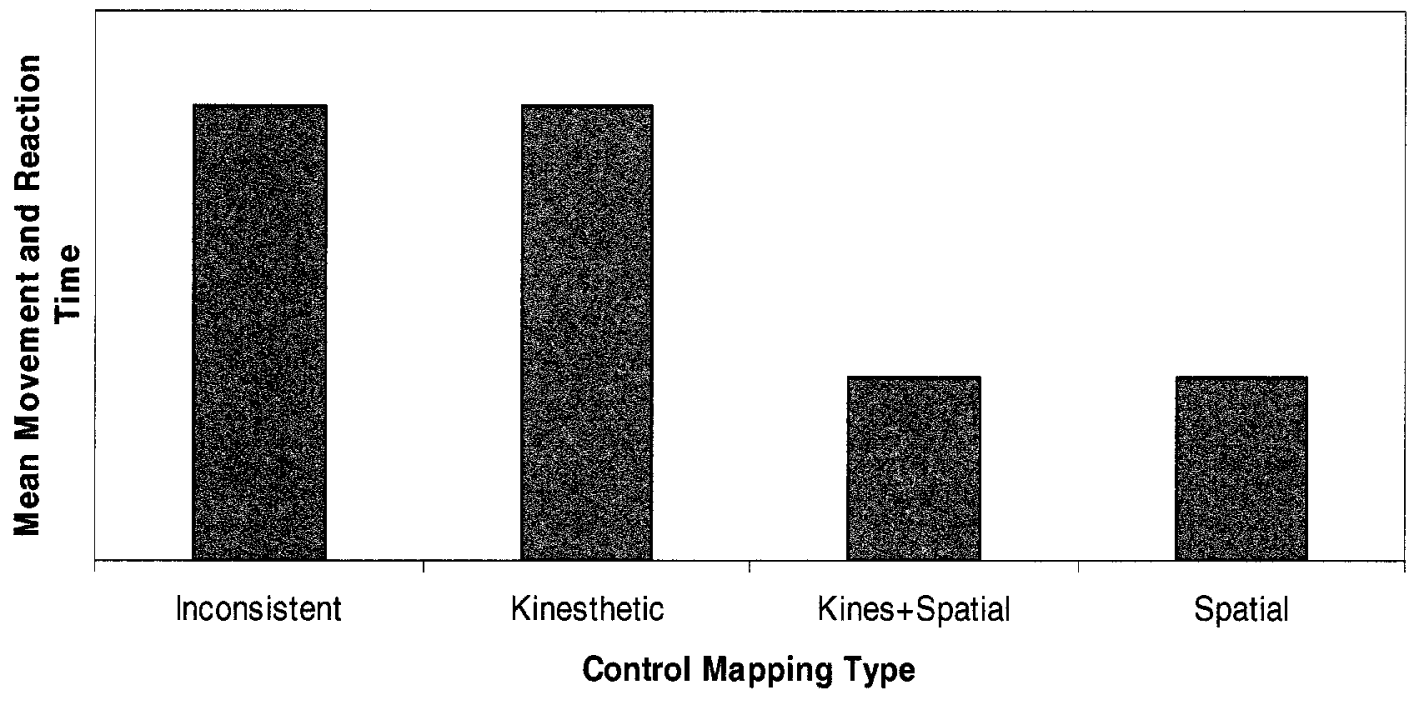

Figure 14. Predicted performance pattern across control mapping type collapsed over symbol type assuming that spatial information has a greater influence. 


\section{METHOD \\ Participants}

Thirty-two participants were recruited from San Jose State University and the NASA Ames Research Center. Participants were required to have $20 / 20$ or corrected to 20/20 vision. Fifty percent of the participants were female and fifty percent were male.

Apparatus and Stimuli

Visual stimuli and test conditions were rendered using a Pentium class desktop PC. The visual stimuli were displayed on a 20" CRT monitor at a resolution of $1280 \mathrm{x}$ 1024. Participants used a 3D Space Mouse rate control to move the displayed symbols (Figure 15). The test room was normally lit at mid-photopic luminance. Participants sat $48.26 \mathrm{~cm}$ from the monitor.

Targets were $3.18 \mathrm{~cm}$ blue squares randomly presented in eight target locations $11.43 \mathrm{~cm}$ from the center of the screen. The cursor was $2.54 \mathrm{~cm}$ in diameter and was either a hand or an arrow. The cursor was presented in one of two orientations (Figure 6). The hand symbol was designed to appear partially grasping so that the image would be more compelling, in an attempt to increase an immersive response to the stimuli. An arrow was used as the compared object because it provided directional cues that would allow for implementation of an implied kinesthetic mapping. To maintain consistency, the arrow was rendered so that it had the same vertical and horizontal asymmetries present in the hand symbol. In Orientation 1, the fingers of the hand symbol pointed away from the participant and were rotated so that the palm faced the left side of the display. When the arrow was in Orientation 1, it was rotated so that the arrow was 


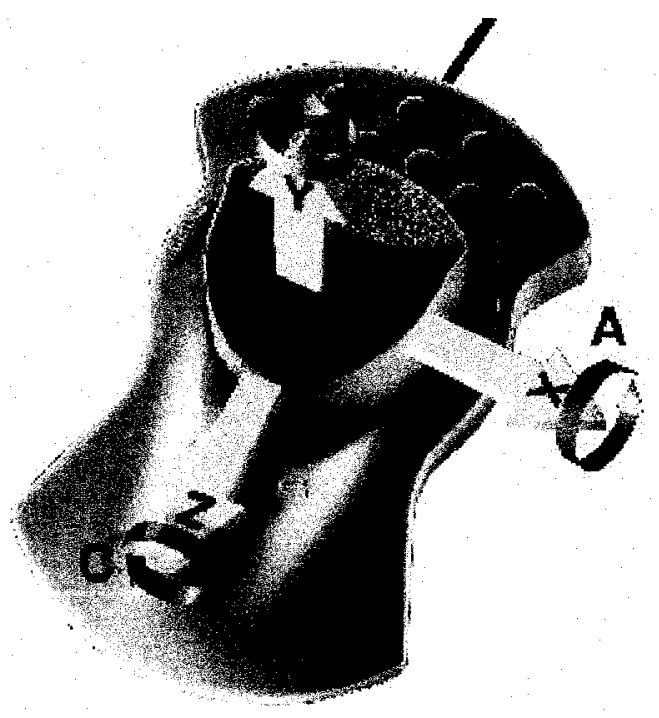

Figure 15. 3D Space Mouse. The 3D Space Mouse provided freedom of movement along the $\mathrm{Y}, \mathrm{X}$, and $\mathrm{Z}$ axis. However, the $\mathrm{Z}$-axis was disabled because the task required only 2D movement. 
horizontally asymmetric such that the left side was colored grey and the right side was colored orange. In Orientation 2, the palm of the hand faced the bottom of the screen and the fingers pointed away from the participant. The orange side of the arrow in Orientation 2 faced the top side of the display.

The initial cursor position was $21.59 \mathrm{~cm}$ from the vertical, and $13.97 \mathrm{~cm}$ from the horizontal edge of the monitor. Movement of the cursor was limited to a 2 dimensional axis. Recall that the purpose of the combined kinesthetic and spatial mapping condition was to provide both spatial and kinesthetic information. In contrast, the kinesthetic mapping condition was created so that the kinesthetic information was isolated. The spatial mapping condition was created to isolate spatial information to a specific mapping configuration. Thus, the Z-axis on the 3D Space Mouse was disabled because we wanted to ensure that all four of the mapping conditions remained mutually exclusive on all possible control movement directions. For example the kinesthetic mapping condition was operationalized by the inverse correspondence between the symbol and the control movement. That is, when the control was moved up and down along the Y-axis, the cursor responded with movements along the horizontal $\mathrm{X}$-axis on the screen. However, enabling the Z-axis would allow participants to move in a direction that would make the correspondence between the symbol and the control consistent, thus violating the operational characteristic of the kinesthetic mapping condition. Movement along the $\mathrm{Z}$-axis in the kinesthetic mapping condition would create symbol and control correspondence that would be characteristic of the combined kinesthetic and spatial mapping condition. 


\section{Control Mapping Conditions}

Participants were given four control mapping configurations, kinesthetic (K), spatial (S), combined kinesthetic + spatial (KS), and inconsistent mapping (I). Control mapping provided kinesthetic information when the direction of the movement of the hand or arrow symbol being controlled was consistent with the movement of the real hand. For example, provided that the hand symbol was in Orientation 1 a heave motion on the Space Mouse corresponded with movement of the cursor along the X-axis. The consistency between the movement of the palm of the real hand on the control and the palm of the hand cursor on the screen suggested that kinesthesia was invoked (Figure 7). Control mapping provided spatial information when the direction of the movement of the hand or arrow symbol being controlled was consistent with movement in space. Figure 8 shows that when the Space Mouse was manipulated in the heave direction, the cursor responded by moving up along the Y-axis. In the KS mapping condition both kinesthetic and spatial mapping corresponded to symbol movement on all the $\mathrm{X}$ and $\mathrm{Y}$ axis. It is important to note that in the KS mapping condition, the hand and arrow were in Orientation 2. This way, when the Space Mouse was moved in the heave direction, the palm of the hand figure and gray side of the arrow remained consistent with the direction of the movement of the palm of the participant's hand (Figure 9). Finally, an inconsistent mapping condition was generated when the movement of the cursor did not correspond kinesthetically or spatially. For example, when the Space Mouse was manipulated in the heave direction and the cursor was in Orientation 2, the cursor responded by moving along the $\mathrm{X}$-axis (Figure 10). Another important note is that the inconsistent mapping 
condition was generated only when the cursors were in Orientation 2. The control mapping was the same in the $\mathrm{K}$ condition, but the symbols were in Orientation 1 .

\section{Design and Procedure}

In this $2 \times 4$ mixed factorial design, the independent variables were symbol type (hand figure vs. arrow) and control mapping type (K, S, KS, I mapping). Symbol type was presented within-subjects. Control mapping type varied between-subjects. Blocks of 80 trials were randomized, each block lasting for a maximum of 80 minutes. Each target location was replicated 10 times. Participants were presented a hand on 80 trials, and an arrow for 80 trials. Hand images were blocked separately from arrow images and counterbalanced. Twenty practice trials for each symbol were given to each participant to help them adapt to the control conditions.

Participants were asked to acquire a target as quickly and accurately as possible, and were only given information about which way they should expect the cursor to move in relationship to the control mapping for the particular condition. For example, if participants were in the $\mathrm{K}$ condition, they were told that if they moved the control horizontally, the cursor would move vertically. During the beginning of each trial, participants fixated on a cross-hair on the center of the screen indicating the future location of the cursor. A trial was successfully completed when the cursor was moved into the square target location and remained in the target for $1 \mathrm{sec}$. Once the target was acquired and the cursor remained in the location for $1 \mathrm{sec}$, the next trial began with crosshairs located at the center of the screen indicating a new trial. In each successive trial the target was located in a random position. Figure 16 illustrates the task sequence. The trial 


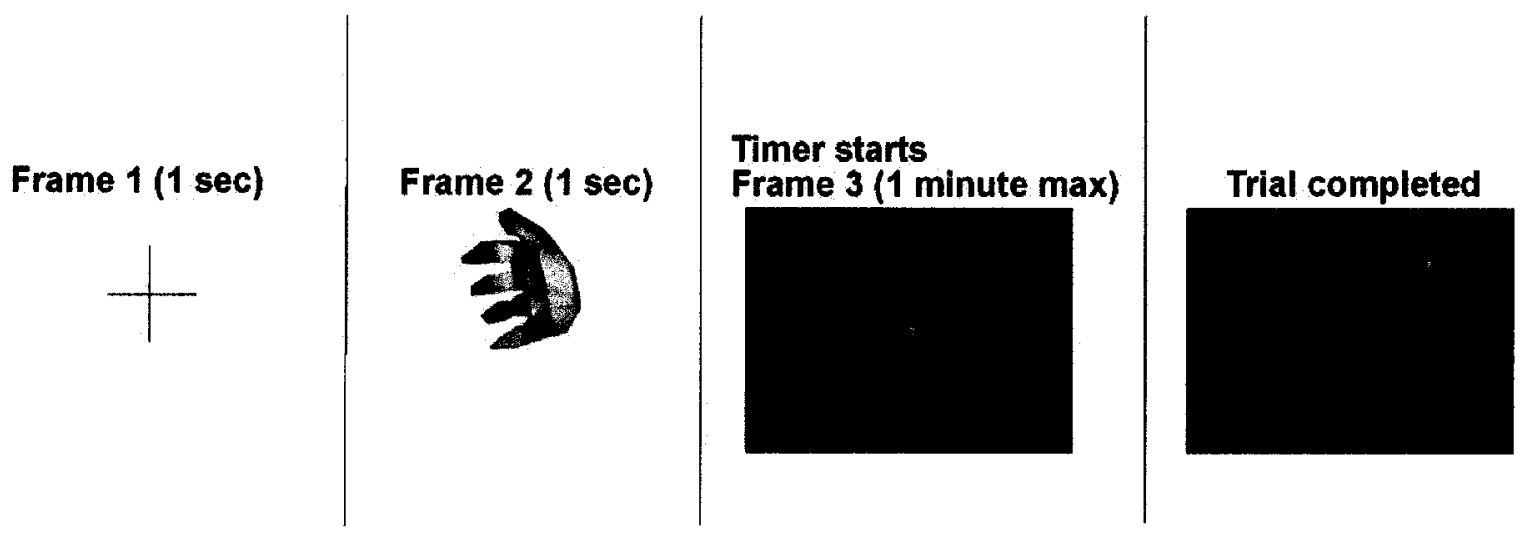

Figure 16. Task Frames. Participants were asked to view the cross hair in Frame 1 for 1 second, after which either the hand of arrow symbol appeared for an additional duration of 1 second. The reaction timer started when the target appeared. The reaction timer stopped and the movement timer started at the on-set of cursor movement. The movement time stopped and the task was completed when the participant maneuvered the symbol inside the blue target and stayed within the target boundary for 1 second. Participants were given a maximum of 1 minute to complete the task. 
timed out if the participant did not successfully complete the task within 1 minute.

Participants did not receive feedback regarding their speed or accuracy. Total duration of the experiment was approximately 1 hour. 


\section{RESULTS}

To test the notion that kinesthetic information can be directly manipulated by varying control mapping configuration, and an "imagined" kinesthesia can be generated by displaying an image of a right-hand as opposed to objects like an arrow, the following hypothesis were formulated. 1.) We did not anticipate a general main effect of symbol type because we reasoned that symbol and control type must interact to generate any kinesthetic information. 2.) Therefore, we expected an interaction effect between symbol and control mapping type, predicting that movement and reaction times will increase or decrease as a function of the control mapping condition. To illustrate the predicted interaction effect, Figure 11 shows that the hand and arrow symbols will elicit comparable performance times in the KS (combined kinesthetic+spatial), and I (inconsistent) conditions. However, the hand symbol was predicted to elicit better performance in the $\mathrm{K}$ (kinesthetic) condition relative to the arrow in the same condition. Inversely, the arrow symbol would elicit better performance in the $S$ (spatial) condition when compared to the hand. 3.) A main effect will be obtained for control mapping type (kinesthetic (K), spatial (S), inconsistent (I), and kinesthetic and spatial combined (KS)), revealing patterns illustrated in Figure 12.

In contrast, if spatial information plays a larger role in manual control performance, another set of hypothesis were generated. 1.) Symbol type will not generate variation in movement or reaction time, thus, no main effect of symbol type is expected if spatial information has greater influence. 2.) No interaction effect will be obtain between control mapping and symbol type because the hand symbol will be perceived as 
an object (arrow) and mean movement, as well as reaction time patterns will coalesce. As shown in Figure 13, performance times for the hand coincided with the arrow regardless of the mapping condition. 3.) Differences in movement and reaction time will persist across control mapping types. However, in the case where spatial information has greater influence, we expect that when movement times for both symbol types are averaged together, slower performance times would be observed for both the inconsistent and kinesthetic mapping conditions. In contrast, both the spatial and the combined kinesthetic and spatial mapping conditions would elicit comparably faster performance times (Figure 14).

\section{Movement Time Data}

To investigate possible differences between control mapping (K, S, I, KS), symbol type (right-hand vs. arrow), as well as possible interaction effects of the control mapping and symbol type, a two-way mixed factorial analysis of variance (ANOVA) was conducted using movement times (in milliseconds) as a dependent variable. Mapping type was the between-subjects factor and symbol type was the within-subjects factor. Movement times were defined by duration of time between the initial movement of the cursor and the placement of the cursor into the target. A significant main effect was found for control mapping type, $\underline{\mathrm{F}}(3,28)=9.42, \underline{\mathrm{p}}<.001$, indicating that movement time data was significantly different among K, I, KS, and S conditions. The post hoc tests revealed that the $\mathrm{I}(M=2414.64)$ and $\mathrm{K}(M=2369.31)$ conditions elicited significantly slower movement times compared to the KS $(M=1008.06)$ and S $(M=1114.19)$ conditions, $\mathfrak{p}<.001$. Additionally, the I and $\mathrm{K}$ conditions did not yield significantly 
different movement time from each other. No main effect was found for symbol type (hand vs. arrow). There was not a significant interaction effect between symbol type and mapping type (Figure 17). At this point, results of the study appear to favor the second set of the aforementioned predictions suggesting that spatial information plays a larger role in manual control performance, and that kinesthetic information did not seem to elicit a strong influence on the current set of data.

Figure 17 shows that mean movement times for the hand symbol appear to be similar to the arrow cursor. However, there seemed to be a greater divergence in mean movement time between the hand and arrow symbol in the kinesthetic and inconsistent mapping condition when compared to other mapping conditions. To investigate this further, other variables which may have suppressed any possible interaction effect of symbol and mapping type were investigated. Thus, practice was analyzed to see if there would be any possible variation in the data pattern.

Participants acquired targets in 8 different locations repeated 10 times for each symbol type (hand and arrow), totaling 80 trials for each symbol, and 160 for a single session. The possible effect of practice was investigated by comparing the first 40 trials with the last 40 trials for each symbol type as within-subjects factor. Trial period (first 40 trials vs. last 40 trials) was included as an additional within-subjects factor. Thus, a 3way mixed factorial ANOVA was conducted. This revealed, again, a main effect for mapping, $\underline{\mathrm{F}}(3,28)=15.301, \mathrm{p}<.001$. Two or three-way interactions were not obtained, and no effect of trial period was found. Practice did not appear to be a confounding variable. 


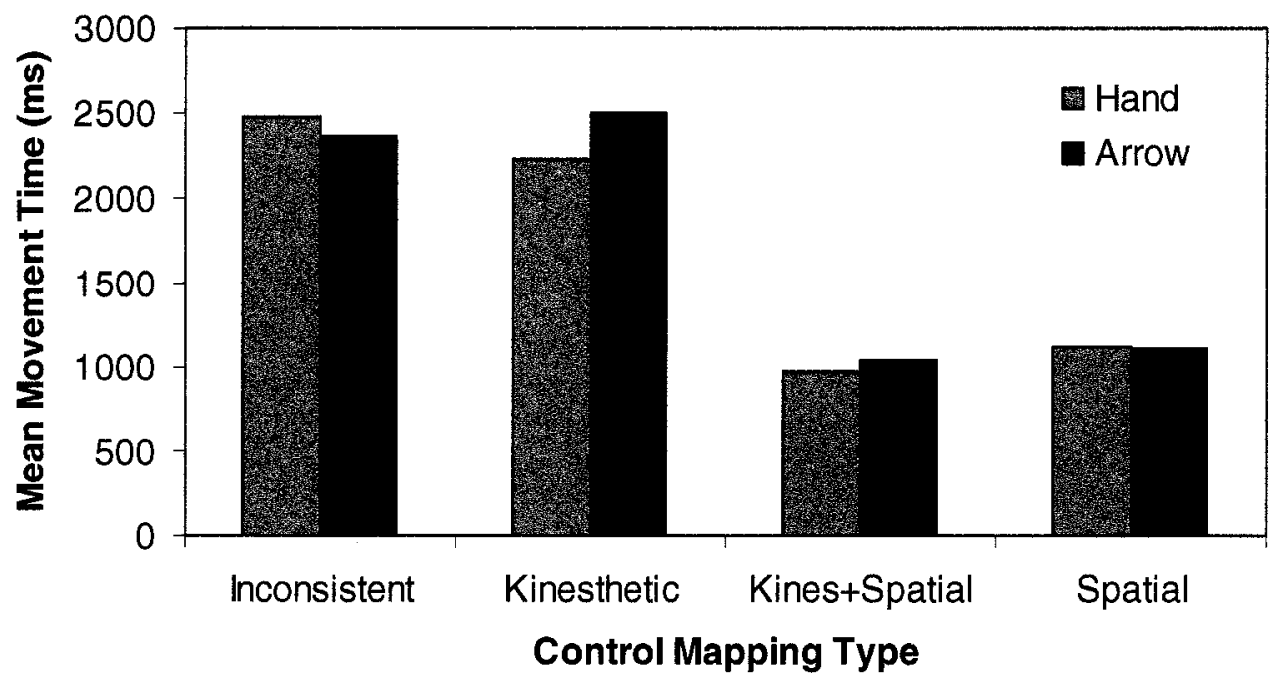

Figure 17. Mean movement times in milliseconds across four mapping conditions for each symbol type. There was no interaction between control mapping type and symbol type. Major movement time differences were due to control/symbol control mapping consistencies. That is, the inconsistent and kinesthetic mapping conditions both had significantly slower movement times when compared to the combined kinesthetic and spatial, and spatial mapping conditions. 


\section{Reaction Time Data}

To investigate pre-motor mental transformation related to our target acquisition task, reaction time measures were also collected. A mixed factorial ANOVA was conducted using target acquisition reaction times as a dependent variable. Reaction times were defined by the duration of time between the initial presentation of the cursor and the onset of the mouse movement. In this mixed design, mapping type was the betweensubjects factor, and symbol type was the within-subjects factor. A significant main effect for control mapping type (K vs. S vs. KS vs. I) was discovered, $\underline{\mathrm{F}}(3,28)=9.42, \underline{\mathrm{p}}<.001$. Similar to the pattern found in the movement time means, in a post hoc analysis of reaction time, performance in the $\mathrm{K}(M=832.21)$ mapping condition was shown to be significantly slower than in the $\mathrm{KS}(M=394.32)$ and $\mathrm{S}(M=428.23)$ conditions, $\mathrm{p}<$ .001. In addition, the I ( $M=700.54)$ mapping condition induced significantly slower reaction times when compared to the $\mathrm{KS}(M=394.32)$ and $S(M=428.23)$ mapping conditions, $\mathrm{p}<.05$ (Figure 14). Again, the $\mathrm{K}$ and I conditions were not significantly different from each other. No main effect was found for symbol type (hand vs. arrow). A two-way interaction for mapping type and symbol type was not found. Thus, it appears that the amount of cognitive effort required to prepare for the target acquisition task at the onset of the presentation of the cursor was analogous to the pattern observed for the mean movement times across each of the mapping conditions.

Similar to patterns found in the movement time data (Figure 17), Figure 18 shows that interaction effects between mapping type and symbol type may have been present between the I (inconsistent) and $\mathrm{K}$ (kinesthetic) mapping conditions. Reaction time data 


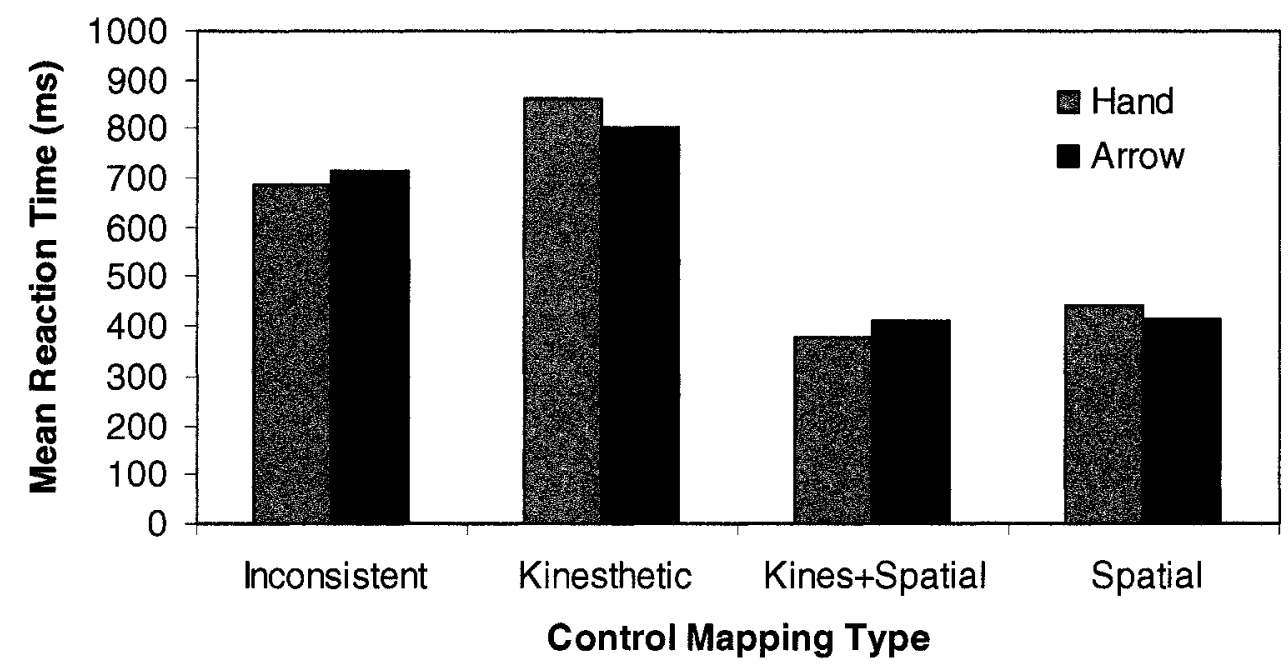

Figure 18. Mean reaction time. Findings for reaction time were similar to those for movement time, again, demonstrating a greater influence of spatial information in manual control performance. 
was separated into early (first 40 trials) and late trial (last 40 trials) periods for each symbol type creating a third between-subjects variable (trial period), and analyzed using three-way mixed factorial ANOVA to see if the effect of symbol may have emerged when looking only at the later trials when participants became accustomed to the control mappings. The absence of an interaction effect of trial period provides evidence that practice did not influence performance times.

Findings from an analysis of movement times and reaction times as dependent variables were similar. A main effect of symbol was not obtained in either of the aforementioned dependent variables. The intervening variable of practice was investigated; but the statistical findings suggested that practice did not play a role. The absence of an interacting symbol and mapping effect, and a symbol type main effect, suggests that the display of hand symbols does not elicit mental transformations that differ from objects such as an arrow. In addition, the findings support the notion that spatial information seems to have a greater influence on manual control performance as predicted and illustrated in Figures 13 and 14.

\section{Self-report Data}

After completion of the experiment, a self-report questionnaire was given to participants to collect information about their age (Figure 19) and perception of the realism of the stimuli, their experience with playing video games, as well as if they had experienced immersion while controlling the hand cursor in the current experiment (Table 1). In general, participants did not perceive the hand symbol as highly realistic. In addition, participants did not feel that the task was immersive, or in other words, they 


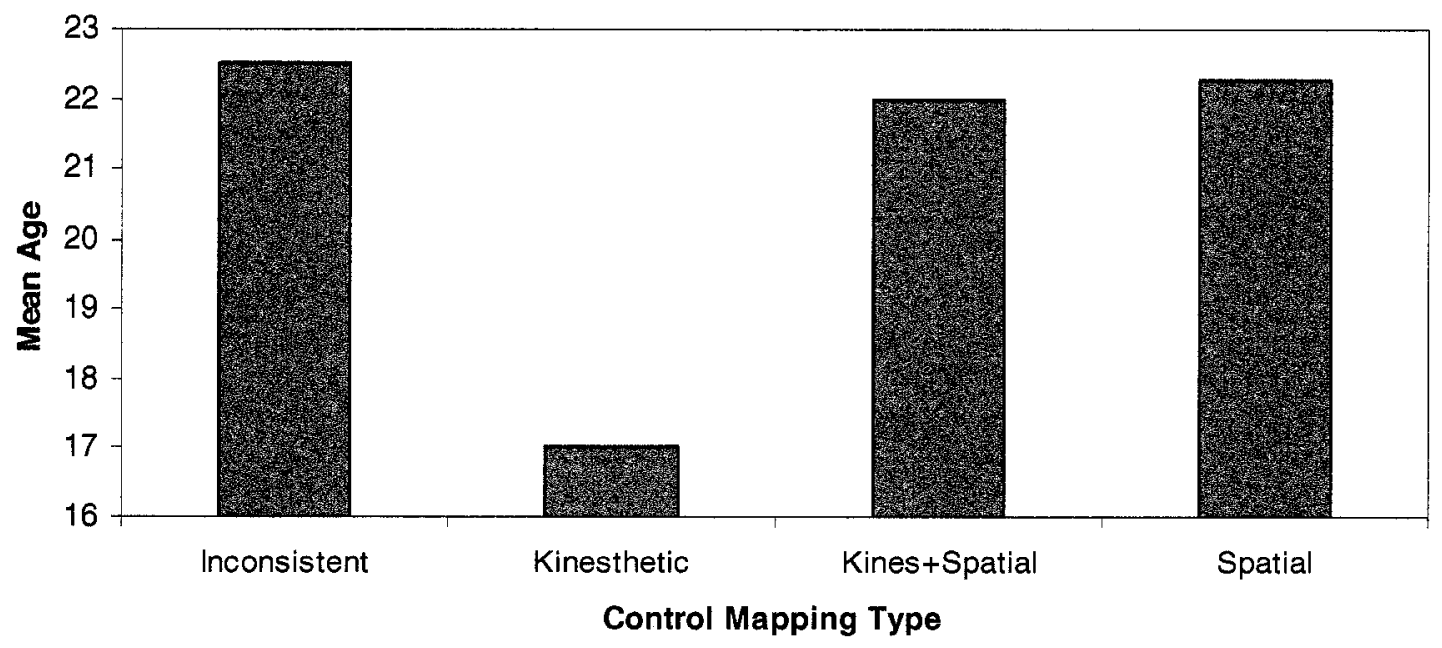

Figure 19. Average age across control mapping conditions. 
Table 1.

Summary of responses collected from participant questionnaire.

\begin{tabular}{llllll}
\hline & & \multicolumn{3}{c}{ Control } \\
& & \multicolumn{3}{c}{ Mapping } \\
\hline Questions & Reply & I & K & KS & S \\
\hline Did the hand look real to you? Rate 1-5 & 1 & 0 & 0 & 0 & 0 \\
& 2 & 3 & 3 & 1 & 0 \\
& 3 & 2 & 4 & 4 & 5 \\
& 4 & 2 & 1 & 1 & 3 \\
& 5 & 1 & 0 & 2 & 0 \\
\hline Did you recognize the arrow? Rate 1-5 & 1 & 0 & 0 & 1 & 1 \\
& 2 & 2 & 1 & 4 & 4 \\
& 3 & 0 & 4 & 0 & 0 \\
& 4 & 5 & 1 & 1 & 3 \\
Did you feel like the hand on the screen was your own? & 5 & 1 & 2 & 2 & 0 \\
\hline Did you feel like you were controlling your own hand? & $\mathrm{Y}$ & 2 & 0 & 3 & 1 \\
& $\mathrm{~N}$ & 6 & 8 & 5 & 7 \\
\hline Have you played 3D immersive video games before? & $\mathrm{Y}$ & 3 & 0 & 3 & 4 \\
& $\mathrm{~N}$ & 5 & 8 & 5 & 4 \\
\hline How often do you play games? Any frequency of time was & $\mathrm{Y}$ & 5 & 4 & 4 & 3 \\
coded Y. Never playing was coded N. & $\mathrm{N}$ & 3 & 4 & 4 & 5 \\
\hline
\end{tabular}


did not feel like they were controlling their own hand as they were attempting to acquire the targets. Therefore, the absence of a main effect for symbol type or a significant interaction with control type, may have been due to the lack of realism in the stimuli. For example, in Table 1, none of the participants in the kinesthetic condition reported that they felt immersed. Fifteen of the 32 participants rated the realism of the hand symbol 3 out of 5 . Twenty-six out of the 32 subjects did not perceive that the hand symbol was their own. Based on the self-report data, we speculate that participants perceived the hand as an object, and preferred the strategy of remembering the spatial relationship between the cursor and the input device. For example, a participant who was given the K condition commented, "I memorized the controls for right and down and thought of the opposite of what I was doing if I was required to go left or up." In contrast, if participants implemented a "kinesthetic" strategy, they might have used the orientation of their thumb as a reference to the direction of movement for the cursor. The spatial nature of the instructions may have had an influence on participants' preference for a spatial strategy to the target acquisition task. The influence of spatial information in the manual control task is evident from the significant findings for control mapping type. Implications of the instructions will be discussed. 


\section{DISCUSSION}

Kinesthesia allows us to complete manual tasks with minimal cognitive effort, effectively reducing response times (McCloskey, 1978). Studies showed that an imagined kinesthesia can be induced with visual stimuli (Sekiyama, 1982). Kinesthetic information can also be directly manipulated to aid manual control performance (Adelstein et al., 2004). In our study we explored the possibility that by eliciting an imagined kinesthesia through visual cueing, as well as by manipulating the mapping on an input device, we can reduce reaction and movement times to reflect the type of response rates in the physical world. We compared reaction time data to investigate premotor processing related to kinesthetic and spatial information conditions. In addition, movement time data was analyzed to see how kinesthetic and spatial information may improve manual control performance. Control mapping type (kinesthetic (K), spatial (S), kinesthetic and spatial combined (KS), and inconsistent (I)) and symbol type (right-hand and arrow) was manipulated to elicit kinesthetic information and spatial information.

We hypothesized that an improvement in the performance for any of the control mapping conditions would be dependent upon the type of symbol presented on the display. That is, we predicted that the hand symbol would elicit faster reaction and movement times in the condition in which kinesthetic information was expected to be present (the $\mathrm{K}$ condition), when compared to the I and $\mathrm{S}$ condition where kinesthetic information was absent. In contrast, we expected that the arrow symbol would lead to faster performance in the $\mathrm{S}$ condition when spatial information was present, when compared to the $\mathrm{K}$ and I conditions. In the condition, where the control mapping 
provided both kinesthetic and spatial information (KS), reaction and movement times were expected to be comparable across the hand and arrow symbols. In the I condition, where there was no spatial or kinesthetic information, reaction and movement times for all symbol types was expected to be slower. In this case, kinesthesia can have an influence on manual control performance provided there is consistent visual and control mapping cues. However, if spatial information had greater influence on manual control performance, the hand symbol would mirror the performance patterns elicited by the arrow symbol. More specifically, in such a case, mean reaction and movement times were predicted to be slow for the I and $\mathrm{K}$ condition, but faster for the KS and $\mathrm{S}$ condition in which object based visual cues are expected to be processed spatially.

Results of our study showed that it was spatial information, rather than kinesthetic information, that had a greater influence on manual control performance. This conclusion was supported by the absence of an interaction effect between symbol type and control mapping type, but a main effect of control type. Reaction and movement times were slower for the control mapping conditions ( $\mathrm{I}$ and $\mathrm{K}$ ) in which the spatial relationship between the control and the cursor was inverted (i.e., horizontal movement on the mouse invoked vertical movement on cursor). However, in the conditions $\mathrm{S}$ and KS where the control mapping was spatially consistent (i.e., horizontal movement on the mouse invoked horizontal movement of the cursor), reaction and movement times were relatively faster. Our findings are consistent with those found in studies conducted by Jordan and Huntsman (1990) leading us to believe that spatial information appears to have a greater influence on manual control performance. 
Although previous research showed that kinesthetic information was manifested in the mental rotation task (Sekiyama, 1982), kinesthetic information did not have an effect on our manual control target acquisition task. Possible reasons for a difference in performance for the kinesthetic condition may be due to the lack of realism in the stimuli. Practice was analyzed, but this factor did not interact with our test conditions. However, self-report data showed that the stimuli generated very low realism with the participants and that the kinesthetic (K) condition was not immersive.

The need for immersion in an "imagined" kinesthetic movement related task was demonstrated in a study conducted by Decety and Jeannerod (1996) where participants were asked to mentally approach a computer generated gate of varying widths. It was found that Fitt's law persisted as participant's verbal report of start to finish times varied with the width of the virtual gate way. It is important to note that participants in the study conducted by Decety and Jeannerod were fully immersed in a 3D scene by wearing a virtual reality helmet. In the current study, only ten of the 32 participants reported that they felt like they were controlling their own hand. None of the participants who were engaged in the $\mathrm{K}$ condition reported feeling like they were controlling their own hand. Three factors may have contributed to the lack of immersion. One of such factors included the inconsistency between the 3D depths cues of the visual stimuli and the $2 \mathrm{D}$ constraints on movement of the target acquisition task. In addition, participants did not report that the hand symbol was highly realistic in appearance. As shown in the selfreport data, 15 participants rated the hand symbol 3 out of 5 for realism. Only 3 thought 
the hand looked very real rating it 5 . Thus, the lack of immersion may have been subdued by the lack of fidelity in the appearance of the hand symbol.

Second, the awkwardness in the acquisition of some of the target locations may have also contributed to the lack of immersion. In the real world, we typically reach for objects with an open hand with fingers outstretched toward the desired object. However, in the present study, participants were forced to acquire targets located behind the palm of the hand symbol since the orientation of the symbol was fixed with the fingers always pointing left. This orientation of the hand cursor may have reduced the immersive effect of the stimuli by creating an awkward and unnatural orientation of the hand symbol when acquiring targets located away from the direction in which the fingers of the hand cursor were pointing. This sense of awkwardness in movement would be consistent with the manageable direction of movement which increased response times for the mental rotation task in Sekiyama's study (1982).

A final factor affecting the experience of immersion in the present study was the nature of the instructions given to participants before execution of the task. The instructions provided to participants were spatial in nature. Participants were only told in what direction they should expect the cursor to move given a particular corresponding direction of movement on the mouse. They were not told to imagine that the hand on the display was their own hand. If participants were told to use their own hand as a reference, it is possible that the movement time may have been faster for the kinesthetic condition in the current study. Evidence for this notion can be found in a study conducted by Cooper and Shepard (1975) where participants were asked to determine if a 
figure of a hand, rotated at varying degrees of misorientation, was left or right. Cooper and Shepard found that when participants were instructed to use their own hand as a reference, response times were faster than when participants were given no instruction. Findings from the current study have direct relevance to the design of control mapping and displays. Mainly, the absence of a main effect for symbol type and no interaction effect of symbol and control mapping, suggested that spatial information played a larger role in our manual control task. In addition, a main effect for control mapping type alone shows that the design of manual control related computer interaction should focus on the spatial relationships between the input device and display elements. In another application, controls for UAV operators should provide spatial cues to maintain exocentric orientation during navigation, instead of using internal references such as kinesthesia.

Future studies examining the role of kinesthesia in manual control performance may include immersion as a factor. Immersion was not manipulated in the current study because data collected for participant experience of immersion was collected only after the experiment was completed. The yes/no responses to an inquiry regarding the experience of immersion were unequally distributed across the different mapping conditions, weakening the validity of the results of any implemented inferential statistics. Instruction can also be manipulated in future studies to see if prior awareness of the presence of kinesthetic information would help to strengthen the kinesthetic effect and the sense of immersion, subsequently improving performance times. In addition, providing awareness that kinesthesia can be used as a cue in our manual control task may 
help to off-set over learned spatial mapping relationships from experience with using ubiquitous standard computer mice. Lastly, an analysis of target location may help to determine if the consistency between simulated hand mediated target acquisition task and the internal representation of natural object acquisition would have an effect on manual control performance. 


\section{REFERENCES}

Adelstein, B. D., Ellis, S. R., Smith, K. D., \& Welch, R. B. (2004). Kinesthetic cueing for manual control in rotated frames: Compact input devices and user technical background. Proceedings of the Human Factors and Ergonomics Society $48^{\text {th }}$ Annual Meeting, 1928-1932.

Bastian, H. C. (1888). The "muscular sense"; its nature and cortical localization. Brain, $10,1-137$.

Britton, E. G., Lipscomb, J. S., \& Pique, M. E. (1978). Making nested rotations convenient for the user. Proceedings of the $5^{\text {th }}$ Annual Conference on Computer Graphics and Interactive Techniques, 12 (3), 222-227.

Cooper, L. A., \& Shepard, R. N. (1973). The time required to prepare for a rotated stimulus. Memory \& Cognition, 6, 98-107.

Cooper, L. A. \& Shepard, R. N. (1975). Mental transformations in the identification of left and right hands. Journal of Experimental Psychology: Human Perception and Performance, 104 (1), 48-56.

Crammond, D. J. (1997). Motor imagery: never in your wildest dream. TINS, 20 (2), 54-57.

Decety, J. \& Jeannerod, M. (1996). Mentally simulated movements in virtual reality: does Fitt's law hold in motor imagery. Behavioural Brain Research, 72, 127-134.

Kosslyn, S. M., Thompson, W. L., Wraga, M., \& Alpter, N. M. (2001). Imagining rotation by endogenous versus exogenous forces: Distinct neural mechanism. Cognitive Neuroscience and Neuropsychology, 12 (11), 2519-2525. 
Jordan, K., \& Huntsman, L. A. (1990). Image rotation of misoriented letter strings: Effects of orientation cuing and repetition. Perception \& Psychophysics, 48 (4), 363-374.

McCloskey, D. I. (1978). Kinesthetic sensibility. Physiological Reviews, 58 (4), 763820.

Sekiyama, K. (1982). Kinesthetic aspects of mental representations in the identification of left and right hands. Perception \& Psychophysics, 32 (2), 89-95.

Wolbers, T., Weiller, C., Buchel, C. (2003). Contralateral coding of imagined body parts in the superior parietal lobe. Cerebral Cortex, 13 (4), 392-399.

Zacks, J. M., Mires, J., Tversky, B. \& Hazeltine, E. (in press). Mental spatial transformations of objects and perspective. Spatial Cognition and Computation, no additional information. 
APPENDIXES 
Appendix A

Consent Form 


\section{Agreement to Participate in Research}

Responsible Investigator(s): Arik-Quang V. Dao

Title of Protocol: The Effect of Kinesthetic and Spatial Information in Manual Control Performance

\section{San José State}

UN|VERSITY

College of Social Sciences

Department of Psychology

One Washington Square

San José, CA 95192-0120

Voice: $408-924-5600$

Fax: 408-924-5605

E-mail: psych@email.sjsu.edu
The California State University: Chancellor's Office

Bakerstield, Channel Islands, Chico

Dominguez Hills, Fresno, Fullerton.

Hayward, Humboldt, Long Beach,

Los Angeles. Maritime Academy,

Monterey Bay, Northridge, Pomona,

Sacramento, San Bernardino, San Diego,

San Francisco, San José, San Luis Oblspo.

Sen Marcos, Sonoma, Stanislaus
1. You have been asked to participate in a research study investigating. The purpose of this study is to examine the role of kinesthetic and spatial information in manual control performance.

2. You will be asked to move a cursor into a target using a Spacemouse controller on a desktop PC. The study will be conducted in the $3^{\text {rd }}$ floor lab in building 262 at the NASA Ames Research Center.

3. There will be no foreseeable risks involved in your participation.

4. There will be no discernable benefits expected from your participation.

\section{5. [Alternative procedures (if applicable). ] NONE}

6. Although the results of this study may be published, no information that could identify you will be included.

7. If you are a student of San Jose State University, you may receive credit toward course work in applicable classes. For all other participants there is no compensation for your participation.

8. Questions about this research may be addressed to Arik-Quang V. Dao (650) 604-6620. Complaints about the research may be presented to Louis Freund, Ph.D Department Chair, Industrial Systems Engineering San Jose State University, (408) 924-3890. Questions about research subjects' rights, or research-related injury may be presented to Pamela Stacks, Ph.D., Interim Associate Vice President, Graduate Studies and Research, at (408) 924-2480.

9. No service of any kind, to which a you are otherwise entitled, will be lost or jeopardized if you choose to "not participate" in the study.

10. Your consent is being given voluntarily. You may refuse to participate in the entire study or in any part of the study. If you decide to participate in the study, you are free to withdraw at any time without any negative effect on your relations with San Jose State University or with any other participating institutions or agencies.

11. At the time that you sign this consent form, you will receive a copy of it for your records, signed and dated by the investigator.

- The signature of a subject on this document indicates agreement to participate in the study.

- The signature of a researcher on this document indicates agreement to include the above named subject in the research and attestation that the subject has been fully informed of his or her rights.

Signature

Date

Investigator's Signature

Date 
Appendix B

Human Subjects Approval Form 


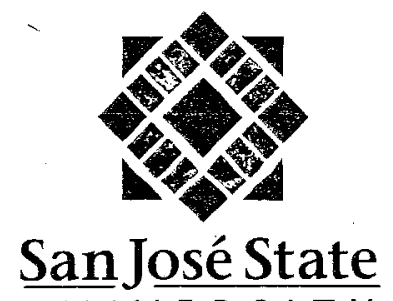

UN IVERSITY

Office of the Academic Vice President

Academic Vice President Graduate Studies and Research

One Washington Square

San José, CA 95192-0025

Voice: $408-283-7500$

Fax: $408-924-2477$

E-mail: gradstudies@sjsu.edu

http://www.sjsu.edu
To: $\quad$ Arik Quang V. Dao 5495 Summerfield Dr. Antioch, CA 94531

From: Pam Stacks,<smiles>C1CCCC1</smiles>

Interim AVP, Graduate Studies \& Research

Date: February 15, 2005

The Human Subjects-Institutional Review Board has approved your request to use human subjects in the study entitled:

"The Effect of Kinesthetic and Spatial Information in Manual Control Performance Abstract."

This approval is contingent upon the subjects participating in your research project being appropriately protected from risk. This includes the protection of the anonymity of the subjects' identity when they participate in your research project, and with regard to all data that may be collected from the subjects. The approval includes continued monitoring of your research by the Board to assure that the subjects are being adequately and properly protected from such risks. If at any time a subject becomes injured or complains of injury, you must notify Pam Stacks, Ph.D. immediately. Injury includes but is not limited to bodily harm, psychological trauma, and release of potentially damaging personal information. This approval for the human subjects portion of your project is in effect for one year, and data collection beyond February 15, 2006 requires an extension request.

Please also be advised that all subjects need to be fully informed and aware that their participation in your research project is voluntary, and that he or she may withdraw from the project at any time. Further, a subject's participation, refusal to participate, or withdrawal will not affect any services that the subject is receiving or will receive at the institution in which the research is being conducted.

If you have any questions, please contact me at (408) 924-2480.

cc: Kevin Jordan, Ph.D. and Kevin Corker, Ph.D. 